Research Article

\title{
Vibration Characteristics Analysis of Convalescent-Wheelchair Robots Equipped with Dynamic Absorbers
}

\author{
Song Wang $(\mathbb{D}$, Leilei Zhao $(\mathbb{D}$, Yanzhu Hu $(\mathbb{D}$, and Fuxing Yang \\ Automation College, Beijing University of Posts and Telecommunications, Beijing 100876, China \\ Correspondence should be addressed to Leilei Zhao; zhaoleilei611571@163.com
}

Received 23 April 2018; Accepted 26 July 2018; Published 24 September 2018

Academic Editor: Angelo Marcelo Tusset

Copyright (c) 2018 Song Wang et al. This is an open access article distributed under the Creative Commons Attribution License, which permits unrestricted use, distribution, and reproduction in any medium, provided the original work is properly cited.

\begin{abstract}
To provide a theoretical guidance for the vibration isolation design, the vibration responses characteristics for the convalescentwheelchair robot with DA (dynamic absorber) undergoing the random road were revealed. Firstly, the vibration model of the convalescent-wheelchair system with DA was created. The frequency response functions of the road excitation velocity to the convalescent acceleration, the wheelchair body acceleration, and the tire dynamic deflection were deduced. Then, the numerical calculation method of the PSD (power spectral density) and the RMS (root mean square) responses were proposed. Thirdly, the vibration isolation performances of the wheelchair robot with DA and without DA were compared. Finally, the sensitivity analysis of the vibration responses to the mass ratios, the damping ratios, and the natural frequencies was carried out to reveal the effects of the parameters on the vibration responses. The results show that the DA can partly suppress the vibration of the convalescent and the wheelchair body, especially in the resonance area of the wheelchair body. However, the DA cannot successfully improve the tire contact behavior.
\end{abstract}

\section{Introduction}

Convalescent-wheelchair robots belong to the rehabilitation medical machines [1]. They are important travel tools for convalescents. With the development of the society and the improvement of the living standard, the demand for them is increasing, for example, their ride comfort, their operational reliability, and so on [2]. When a wheelchair robot runs on the random road, its vibration caused by the road irregularity is inevitable. The harmful vibration produces adverse effects for the wheelchair and its users [3-5].

In recent years, many scholars have studied the related vibration problems of wheelchair robots. The damping characteristics of the wheelchair cushion were studied on the basis of the standard ISO 16840-2:2007 in [6]. A new dynamic model for the wheelchair coupled with the user was developed and validated in [7]. The vibration transmission characteristics of wheelchairs and their effects on patients were analyzed in [8]. Hikmawan and Nugraha [9] simulated the vibration of a wheelchair under the sinusoidal excitation based on a 6 degree of freedom model of the wheelchair user. $\mathrm{Wu}$ et al. [10] proposed a new method of evaluating the ride comfort of wheelchair robots based on the Q-learning algorithm, which has a certain reference value for the vibration evaluation of wheelchair robots. Wang et al. [11] established a mathematical model of the wheelchair cushion comfortableness, which provides a reference for the wheelchair design. Miyawaki and Takahashi [12] designed a new wheelchair and analyzed the whole-body vibration of the user sitting on the wheelchair. Su et al. [13] designed a magnetic suspension vibrator to improve the vibration attenuation performance of electric wheelchairs. Ababou et al. [14] developed a test bench to provide harmful vibrations for wheelchairs and the test bench can be applied to analyze the wheelchair transmissibility. Kundu et al. [15] designed a four wheel-driven wheelchair and investigated its sliding and vibration characteristics. Garcia-Mendenz et al. [16] estimated the whole-body vibration of the wheelchair users in their communities. Requejo et al. [17] studied the effect of hand-rim wheelchairs equipped with rear 
suspension on seat forces. The influences of the user anatomy and wheelchair cushion type on the tissue deformation were analyzed in [18]. The aforementioned studies provide useful references for improving the vibration isolation performances of wheelchair robots. However, there are few studies on the comprehensive performance of wheelchair robots undergoing the random road.

Most of convalescent-wheelchair robots do not have suspension systems due to the limited installation space. Their users' vibration caused by the road excitation is mainly reduced by the tires and the cushion. The DA is a vibration reducing device connected to the vibration system by elastic and damping elements $[19,20]$. It occupies a small space and provides a cheap and effective way to attenuate the wheelchair robots vibration.

In the previous work [21], the impact responses and parameters sensitivity of electric wheelchairs were analyzed. In this paper, to provide a theoretical support for the design of convalescent-wheelchair robots, the vibration responses characteristics for the convalescent-wheelchair robot with DA undergoing the random road were revealed.

\section{The Vibration Model of the Convalescent- Wheelchair System with DA}

In the initial design stage of convalescent-wheelchair robots, the parameters needed to establish accurate vibration models are often unknown. Therefore, to easily guide their design in theory, the convalescent-wheelchair system should be simplified.

In this paper, the cushion and tires are equivalent to a spring-damper, respectively. Moreover, the convalescent is regarded as a rigid body mass. The vibration model of the convalescent-wheelchair system with DA is created, as shown in Figure 1 [21]. In Figure $1, m_{2}, m_{1}$, and $m_{0}$ represent the convalescent mass, the wheelchair body mass, and the DA mass, respectively; $K_{2}, K_{1}$, and $K_{0}$ represent the cushion stiffness, the tire stiffness, and the DA stiffness, respectively; $C_{2}, C_{1}$, and $C_{0}$ represent the cushion damping, the tire damping, and the DA damping, respectively; $z_{2}, z_{1}$, and $z_{0}$ represent the convalescent vertical displacement, the wheelchair body vertical displacement, and the DA vertical displacement, respectively; and $q$ represents the road displacement input. To facilitate the comparative analysis, the vibration model of the convalescent-wheelchair system without DA is also given in Figure 2.

According to Newton's second law, the motion equation for the vibration model of the convalescent-wheelchair system without DA can be expressed as

$$
\left\{\begin{array}{l}
m_{2} \ddot{z}_{2}+C_{2}\left(\dot{z}_{2}-\dot{z}_{1}\right)+K_{2}\left(z_{2}-z_{1}\right)=0 \\
m_{1} \ddot{z}_{1}+C_{2}\left(\dot{z}_{1}-\dot{z}_{2}\right)+K_{2}\left(z_{1}-z_{2}\right)+C_{1}\left(\dot{z}_{1}-\dot{q}\right)+K_{1}\left(z_{1}-q\right)=0 .
\end{array}\right.
$$

Analogously, the motion equation for the vibration model of the convalescent-wheelchair system with DA can be expressed as

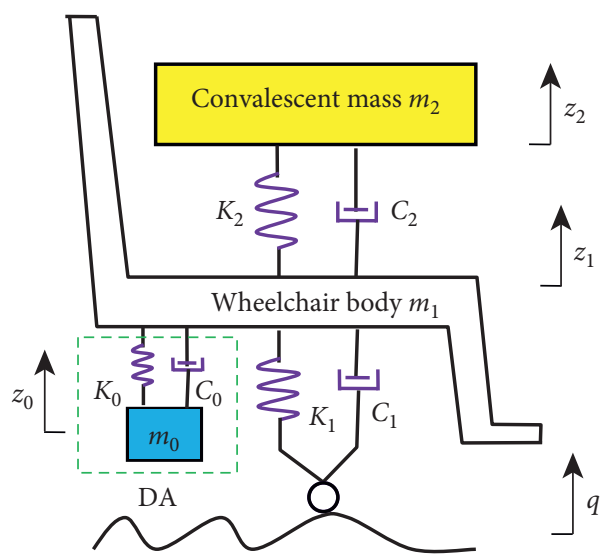

FIgURE 1: The vibration model of the convalescent-wheelchair system with DA.

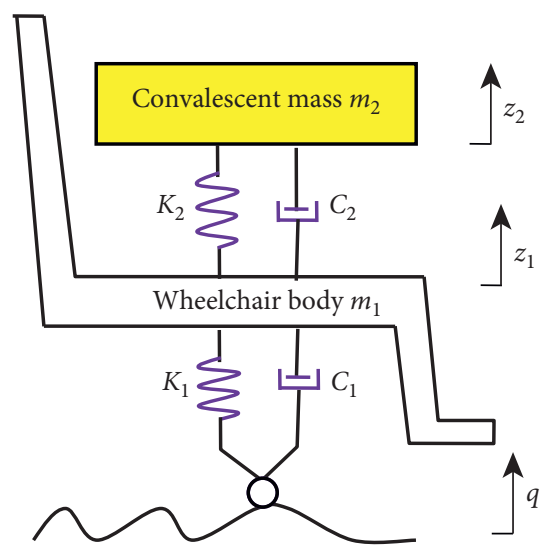

FIgURE 2: The vibration model of the convalescent-wheelchair system without DA.

$$
\left\{\begin{array}{l}
m_{2} \ddot{z}_{2}+C_{2}\left(\dot{z}_{2}-\dot{z}_{1}\right)+K_{2}\left(z_{2}-z_{1}\right)=0, \\
m_{0} \ddot{z}_{0}+C_{0}\left(\dot{z}_{0}-\dot{z}_{1}\right)+K_{0}\left(z_{0}-z_{1}\right)=0, \\
m_{1} \ddot{z}_{1}+C_{2}\left(\dot{z}_{1}-\dot{z}_{2}\right)+K_{2}\left(z_{1}-z_{2}\right)+C_{0}\left(\dot{z}_{1}-\dot{z}_{0}\right) \\
\quad+K_{0}\left(z_{1}-z_{0}\right)+C_{1}\left(\dot{z}_{1}-\dot{q}\right)+K_{1}\left(z_{1}-q\right)=0 .
\end{array}\right.
$$

By comparing Equation (1) with Equation (2), when $m_{0}$ equals zero, Equation (2) changes into Equation (1).

To easily facilitate the wheelchair robots design, the following auxiliary variables are introduced:

$$
\begin{aligned}
& r_{0}=\frac{m_{0}}{m_{1}}, \\
& r_{2}=\frac{m_{2}}{m_{1}}, \\
& f_{2}=\frac{1}{2 \pi} \sqrt{\frac{K_{2}}{m_{2}},} \\
& f_{1}=\frac{1}{2 \pi} \sqrt{\frac{K_{1}}{m_{1}}},
\end{aligned}
$$




$$
\begin{aligned}
& f_{0}=\frac{1}{2 \pi} \sqrt{\frac{K_{0}}{m_{0}}}, \\
& \xi_{2}=\frac{C_{2}}{2 \sqrt{m_{2} K_{2}}}, \\
& \xi_{1}=\frac{C_{1}}{2 \sqrt{m_{1} K_{1}}}, \\
& \xi_{0}=\frac{C_{0}}{2 \sqrt{m_{0} K_{0}}},
\end{aligned}
$$

where, $r_{0}$ and $r_{2}$ are the mass ratios; $f_{0}$ is defined as the DA natural frequency; $f_{1}$ is defined as the wheelchair body natural frequency; $f_{2}$ is defined as the convalescent natural frequency; and $\xi_{0}, \xi_{1}$, and $\xi_{2}$ are the damping ratios of the DA system, the tire system, and the cushion system.

Based on the introduced variables, Equation (2) can be expressed as

$$
\left\{\begin{array}{l}
m_{2} \ddot{z}_{2}+2 m_{2} \xi_{2} \omega_{2}\left(\dot{z}_{2}-\dot{z}_{1}\right)+m_{2} \omega_{2}^{2}\left(z_{2}-z_{1}\right)=0 \\
m_{0} \ddot{z}_{0}+2 m_{0} \xi_{0} \omega_{0}\left(\dot{z}_{0}-\dot{z}_{1}\right)+m_{0} \omega_{0}^{2}\left(z_{0}-z_{1}\right)=0 \\
m_{1} \ddot{z}_{1}+2 m_{2} \xi_{2} \omega_{2}\left(\dot{z}_{1}-\dot{z}_{2}\right)+m_{2} \omega_{2}^{2}\left(z_{1}-z_{2}\right)+2 m_{0} \xi_{0} \omega_{0}\left(\dot{z}_{1}-\dot{z}_{0}\right) \\
\quad+m_{0} \omega_{0}^{2}\left(z_{1}-z_{0}\right)+2 m_{1} \xi_{1} \omega_{1}\left(\dot{z}_{1}-\dot{q}\right)+m_{1} \omega_{1}^{2}\left(z_{1}-q\right)=0,
\end{array}\right.
$$

where, $\omega_{0}=2 \pi f_{0}, \omega_{1}=2 \pi f_{1}$, and $\omega_{2}=2 \pi f_{2}$.

Equation (4) can be simplified as

$$
\left\{\begin{array}{l}
\ddot{z}_{2}+2 \xi_{2} \omega_{2}\left(\dot{z}_{2}-\dot{z}_{1}\right)+\omega_{2}^{2}\left(z_{2}-z_{1}\right)=0, \\
\ddot{z}_{0}+2 \xi_{0} \omega_{0}\left(\dot{z}_{0}-\dot{z}_{1}\right)+\omega_{0}^{2}\left(z_{0}-z_{1}\right)=0, \\
\ddot{z}_{1}+2 r_{2} \xi_{2} \omega_{2}\left(\dot{z}_{1}-\dot{z}_{2}\right)+r_{2} \omega_{2}^{2}\left(z_{1}-z_{2}\right)+2 r_{0} \xi_{0} \omega_{0}\left(\dot{z}_{1}-\dot{z}_{0}\right) \\
\quad+r_{0} \omega_{0}^{2}\left(z_{1}-z_{0}\right)+2 \xi_{1} \omega_{1}\left(\dot{z}_{1}-\dot{q}\right)+\omega_{1}^{2}\left(z_{1}-q\right)=0 .
\end{array}\right.
$$

\section{System Amplitude Frequency Characteristics}

For the free vibration of the convalescent-wheelchair system with DA, each mass vibrates at the same circular frequency $\omega$ and the same phase angle $\varphi$. Moreover, the corresponding amplitudes are $z_{00}, z_{10}$, and $z_{20}$, respectively. Thus, the solution of the free vibration equation can be expressed as

$$
\left\{\begin{array}{l}
z_{0}=z_{00} e^{j(\omega t+\varphi)}, \\
z_{1}=z_{10} e^{j(\omega t+\varphi)}, \\
z_{2}=z_{20} e^{j(\omega t+\varphi)} .
\end{array}\right.
$$

Substituting Equation (6) to Equation (5) produces

$$
\left\{\begin{array}{l}
z_{2}\left(-\omega^{2}+2 \xi_{2} \omega_{2} \omega j+\omega_{2}^{2}\right)=z_{1}\left(2 \xi_{2} \omega_{2} \omega j+\omega_{2}^{2}\right), \\
z_{0}\left(-\omega^{2}+2 \xi_{0} \omega_{0} \omega j+\omega_{0}^{2}\right)=z_{1}\left(2 \xi_{0} \omega_{0} \omega j+\omega_{0}^{2}\right), \\
z_{1}\left[-\omega^{2}+\left(2 r_{0} \xi_{0} \omega_{0}+2 \xi_{1} \omega_{1}\right) \omega j+r_{2} \omega_{2}^{2}+r_{0} \omega_{0}^{2}+\omega_{1}^{2}\right] \\
=z_{2}\left(2 r_{2} \xi_{2} \omega_{2} \omega j+r_{2} \omega_{2}^{2}\right)+z_{0}\left(2 r_{0} \xi_{0} \omega_{0} \omega j+r_{0} \omega_{0}^{2}\right) \\
\quad+q\left(2 \xi_{1} \omega_{1} \omega j+\omega_{1}^{2}\right) .
\end{array}\right.
$$

Equation (7) can be further expressed as

$$
\left\{\begin{array}{l}
A_{1} z_{2}=A_{2} z_{1}, \\
A_{3} z_{0}=A_{4} z_{1}, \\
A_{5} z_{1}=A_{6} z_{2}+A_{7} z_{0}+A_{8} q .
\end{array}\right.
$$

where

$$
\begin{aligned}
A_{1}= & -\omega^{2}+2 \xi_{2} \omega_{2} \omega j+\omega_{2}^{2}, \\
A_{2}= & 2 \xi_{2} \omega_{2} \omega j+\omega_{2}^{2}, \\
A_{3}= & -\omega^{2}+2 \xi_{0} \omega_{0} \omega j+\omega_{0}^{2}, \\
A_{4}= & 2 \xi_{0} \omega_{0} \omega j+\omega_{0}^{2}, \\
A_{5}= & -\omega^{2}+\left(2 r_{0} \xi_{0} \omega_{0}+2 r_{2} \xi_{2} \omega_{2}+2 \xi_{1} \omega_{1}\right) \omega j \\
& +r_{2} \omega_{2}^{2}+r_{0} \omega_{0}^{2}+\omega_{1}^{2}, \\
A_{6}= & 2 r_{2} \xi_{2} \omega_{2} \omega j+r_{2} \omega_{2}^{2}, \\
A_{7}= & 2 r_{0} \xi_{0} \omega_{0} \omega j+r_{0} \omega_{0}^{2}, \\
A_{8}= & 2 \xi_{1} \omega_{1} \omega j+\omega_{1}^{2} .
\end{aligned}
$$

From Equation (8), obtain the following:

$$
\begin{aligned}
& \frac{z_{2}}{q}=\frac{A_{2} A_{3} A_{8}}{A_{1} A_{3} A_{5}-A_{2} A_{3} A_{6}-A_{1} A_{4} A_{7}}, \\
& \frac{z_{1}}{q}=\frac{A_{1} A_{3} A_{8}}{A_{1} A_{3} A_{5}-A_{2} A_{3} A_{6}-A_{1} A_{4} A_{7}} .
\end{aligned}
$$

The amplitude frequency characteristics function $J_{1}$ between the road velocity input $\dot{q}$ and the wheelchair body acceleration $\ddot{z}_{1}$ can be expressed as

$$
J_{1}=|H(j \omega)|_{\ddot{z}_{1} \sim \dot{q}}=\left|\frac{\ddot{z}_{1}}{\dot{q}}\right|=\omega\left|\frac{z_{1}}{q}\right| .
$$

The amplitude frequency characteristics function $J_{2}$ between the road velocity input $\dot{q}$ and the convalescent acceleration $\ddot{z}_{2}$ can be expressed as

$$
J_{2}=|H(j \omega)|_{\ddot{z}_{2} \sim \dot{q}}=\left|\frac{\ddot{z}_{2}}{\dot{q}}\right|=\omega\left|\frac{z_{2}}{q}\right| .
$$

The amplitude frequency characteristics function $J_{3}$ between the road velocity input $\dot{q}$ and the TDD (tire dynamic deflection) $f_{\mathrm{d}}=z_{1}-q$ can be expressed as 


$$
J_{3}=|H(j \omega)|_{f_{\mathrm{d}} \sim \dot{q}}=\left|\frac{f_{\mathrm{d}}}{\dot{q}}\right|=\left|\frac{z_{1}-q}{\dot{q}}\right|=\frac{1}{\omega}\left|\frac{z_{1}-q}{q}\right| .
$$

Substituting Equation (11) in Equation (12) produces

$$
J_{1}=\omega\left|\frac{A_{1} A_{3} A_{8}}{A_{1} A_{3} A_{5}-A_{2} A_{3} A_{6}-A_{1} A_{4} A_{7}}\right| .
$$

Substituting Equation (10) in Equation (13) produces

$$
J_{2}=\omega\left|\frac{A_{2} A_{3} A_{8}}{A_{1} A_{3} A_{5}-A_{2} A_{3} A_{6}-A_{1} A_{4} A_{7}}\right| .
$$

Substituting Equation (11) in Equation (15) produces

$$
J_{3}=\frac{1}{\omega}\left|\frac{A_{1} A_{3} A_{8}-A_{1} A_{3} A_{5}+A_{2} A_{3} A_{6}+A_{1} A_{4} A_{7}}{A_{1} A_{3} A_{5}-A_{2} A_{3} A_{6}-A_{1} A_{4} A_{7}}\right| \text {. }
$$

\section{The PSD and RMS Responses under the Random Road}

4.1. Random Road Model. The spatial frequency PSD $G_{q}(n)$ of the random road roughness $q$ can be expressed as [22]

$$
G_{q}(n)=G_{q}\left(n_{0}\right)\left(\frac{n}{n_{0}}\right)^{-w},
$$

where, $n$ is the spatial frequency; $n_{0}$ is the spatial reference frequency and $n_{0}=0.1 \mathrm{~m}^{-1} ; G_{q}\left(n_{0}\right)$ is the road roughness coefficient; and $w$ is the frequency index.

When a wheelchair robot runs on the random road at a certain speed $u$, the time frequency of the road excitation is $f=u n$. Thus, the time frequency PSD $G_{q}(f)$ is

$$
G_{q}(f)=\frac{1}{u} G_{q}(n)
$$

When $w=2$, the time frequency $\operatorname{PSD} G_{q}(f)$ can be expressed as

$$
G_{q}(f)=\frac{1}{u} G_{q}\left(n_{0}\right)\left(\frac{n}{n_{0}}\right)^{-2}=G_{q}\left(n_{0}\right) n_{0}^{2} \frac{u}{f^{2}} .
$$

The relationship between the time frequency PSD $G_{q}(f)$ of the random road roughness $q$ and the time frequency PSD $G_{\dot{q}}(f)$ of the random road excitation velocity $\dot{q}$ is given by

$$
G_{\dot{q}}(f)=(2 \pi f)^{2} G_{q}(f)=4 \pi^{2} G_{q}\left(n_{0}\right) n_{0}^{2} u \text {. }
$$

4.2. The PSD and RMS Responses Calculation. The autocorrelation function $R_{\dot{q}}(\tau)$ of $\dot{q}(t)$ is

$$
R_{\dot{q}}(\tau)=\lim _{T \longrightarrow \infty} \frac{1}{T} \int_{-T / 2}^{+T / 2} \dot{q}(t) \dot{q}(t+\tau) d t .
$$

The convalescent acceleration $\ddot{z}_{2}(t)$ can be expressed as

$$
\ddot{z}_{2}(t)=\int_{0}^{+\infty} \dot{q}(\tau) h(t-\tau) d \tau
$$

where $h(t)$ is impulse response function.

The PSD $G_{\ddot{z}_{2}}(\omega)$ of the convalescent acceleration $\ddot{z}_{2}(t)$ can be expressed as
TABLE 1: The values of the parameters for the convalescentwheelchair system.

\begin{tabular}{lc}
\hline Parameter & Value \\
\hline$m_{2}(\mathrm{~kg})$ & 75 \\
$m_{1}(\mathrm{~kg})$ & 25 \\
$m_{0}(\mathrm{~kg})$ & 2.5 \\
$C_{2}(\mathrm{Ns} / \mathrm{m})$ & 377 \\
$C_{1}(\mathrm{Ns} / \mathrm{m})$ & 314 \\
$C_{0}(\mathrm{Ns} / \mathrm{m})$ & 63 \\
$K_{2}(\mathrm{~N} / \mathrm{mm})$ & 11.84 \\
$K_{1}(\mathrm{~N} / \mathrm{mm})$ & 98.70 \\
$K_{0}(\mathrm{~N} / \mathrm{mm})$ & 9.87 \\
\hline
\end{tabular}

$$
G_{\ddot{z}_{2}}(\omega)=E\left[\ddot{z}_{2}(t) \ddot{z}_{2}(t+\tau)\right]=H(j \omega) G_{\dot{q}}(\omega) H(j \omega)=J^{2} G_{\dot{q}}(\omega) .
$$

Substituting Equation (21) in Equation (24) produces

$$
G_{\ddot{z}_{2}}(\omega)=4 \pi^{2} G_{q}\left(n_{0}\right) n_{0}^{2} u J_{2}^{2} \text {. }
$$

The convalescent RMS acceleration $\sigma_{\ddot{z}_{2}}$ can be expressed as

$$
\sigma_{\ddot{z}_{2}}=\sqrt{\frac{1}{2 \pi} \int_{0}^{+\infty} G_{\ddot{z}_{2}}(\omega) d \omega}
$$

Substituting Equation (25) in Equation (26) produces

$$
\sigma_{\ddot{z}_{2}}=\sqrt{2 \pi G_{q}\left(n_{0}\right) n_{0}^{2} u \int_{0}^{+\infty} J_{2}^{2}(\omega) d \omega} .
$$

Analogously, the wheelchair body RMS acceleration $\sigma_{\ddot{z}_{1}}$ can be expressed as

$$
\sigma_{\ddot{z}_{1}}=\sqrt{2 \pi G_{q}\left(n_{0}\right) n_{0}^{2} u \int_{0}^{+\infty} J_{1}^{2}(\omega) d \omega} .
$$

The TDD RMS can be expressed as

$$
\sigma_{f_{\mathrm{d}}}=\sqrt{2 \pi G_{q}\left(n_{0}\right) n_{0}^{2} u \int_{0}^{+\infty} J_{3}^{2}(\omega) d \omega} .
$$

It is difficult to analytically calculate the Equations (27)-(29). The numerical integration method is adopted as

$$
\begin{gathered}
\sigma_{\ddot{z}_{2}}=\sqrt{2 \pi G_{q}\left(n_{0}\right) n_{0}^{2} u \sum_{i=1}^{N}\left[J_{2}^{2}(2 \pi i \Delta f)\right] \times(2 \pi i \Delta f),} \\
\sigma_{\ddot{z}_{1}}=\sqrt{2 \pi G_{q}\left(n_{0}\right) n_{0}^{2} u \sum_{i=1}^{N}\left[J_{1}^{2}(2 \pi i \Delta f)\right] \times(2 \pi i \Delta f),} \\
\sigma_{f_{\mathrm{d}}}=\sqrt{2 \pi G_{q}\left(n_{0}\right) n_{0}^{2} u \sum_{i=1}^{N}\left[J_{3}^{2}(2 \pi i \Delta f)\right] \times(2 \pi i \Delta f)},
\end{gathered}
$$

where $N$ is the number of the discrete frequency value; $\Delta f$ is the frequency bandwidth; and $i=1,2,3, \ldots, N$. 


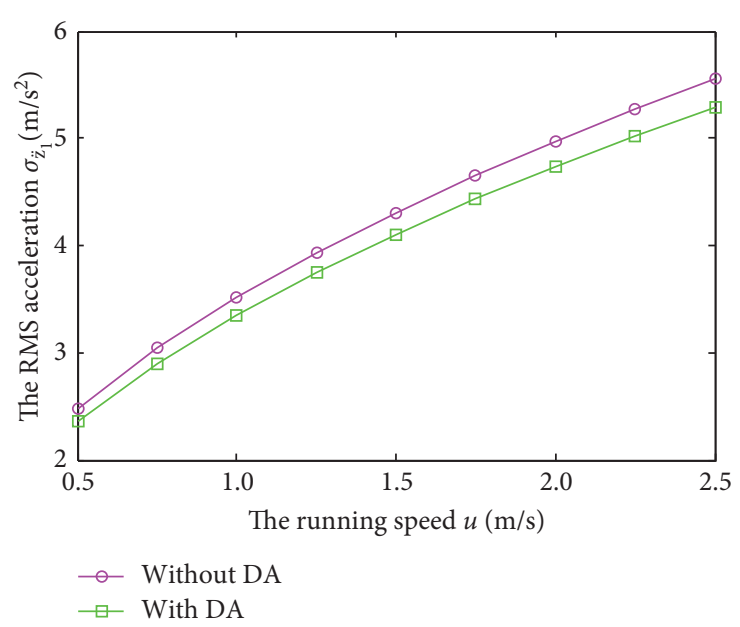

(a)

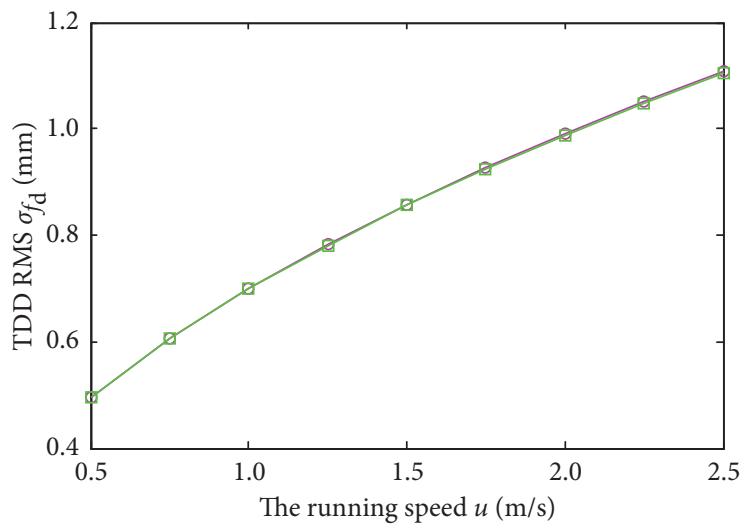

$\rightarrow$ Without DA

$\neg$ With DA

(c)

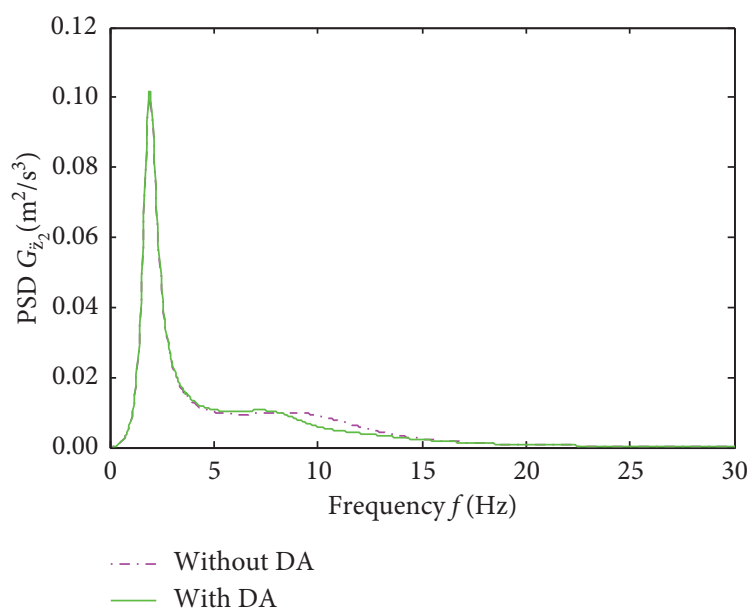

(e)

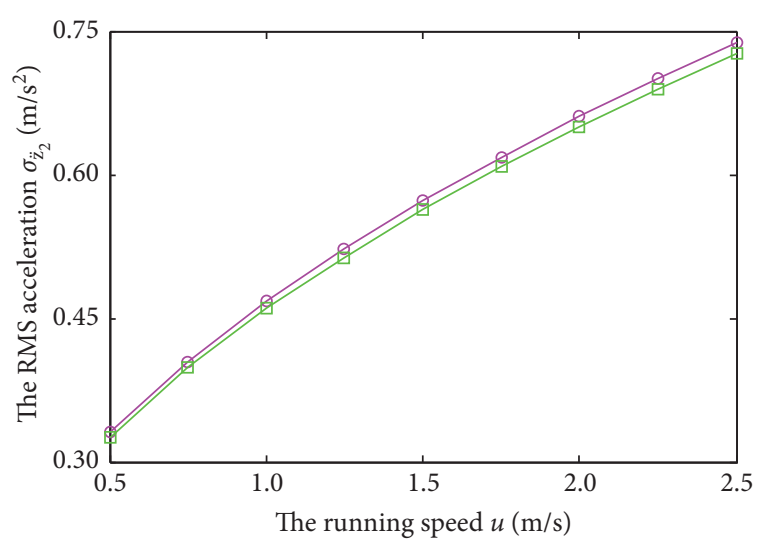

- Without DA

$\square$ With DA

(b)

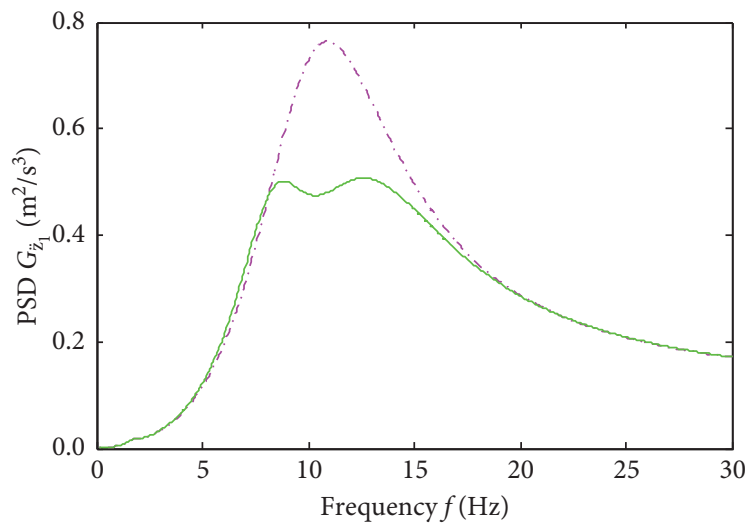

-..- Without DA

With DA

(d)

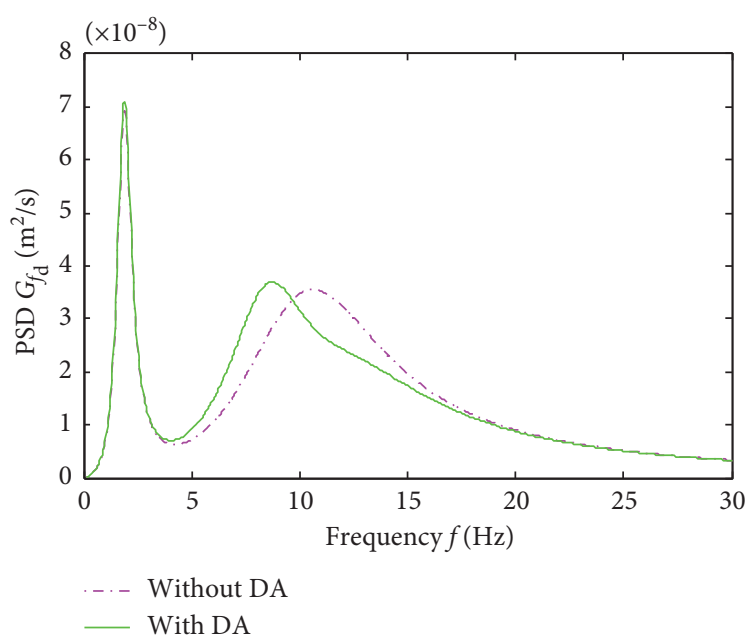

(f)

FIgURE 3: A comparison of the dynamic responses calculated from the vibration models of the convalescent-wheelchair with DA and without DA: (a) the wheelchair body RMS acceleration $\sigma_{\ddot{z}_{1}}$ versus the running speed $u$; (b) the convalescent RMS acceleration $\sigma_{\ddot{z}_{2}}$ versus the running speed $u$; (c) the TDD RMS $\sigma_{f_{\mathrm{d}}}$ versus the running speed $u$; (d) the wheelchair body acceleration PSD $G_{\ddot{z}_{1}}$ versus the frequency $f$; (e) the convalescent acceleration PSD $G_{\ddot{z}_{2}}$ versus the frequency $f$; (f) the TDD PSD $G_{f_{\mathrm{d}}}$ versus the frequency $f$. 
TABLE 2: The values of the system parameters for the analysis.

\begin{tabular}{lccc}
\hline Parameter & Baseline & $+100 \%$ & $-50 \%$ \\
\hline$r_{2}$ & 3.0 & 6.0 & 1.5 \\
$r_{0}$ & 0.10 & 0.05 & 0.20 \\
$\xi_{2}$ & 0.25 & 0.5 & 0.125 \\
$\xi_{1}$ & 0.25 & 0.5 & 0.125 \\
$\xi_{0}$ & 0.25 & 0.5 & 0.125 \\
$f_{2}$ & 2.0 & 4.0 & 1.0 \\
$f_{1}$ & 10.0 & 20.0 & 5.0 \\
$f_{0}$ & 10.0 & 20.0 & 5.0 \\
\hline
\end{tabular}

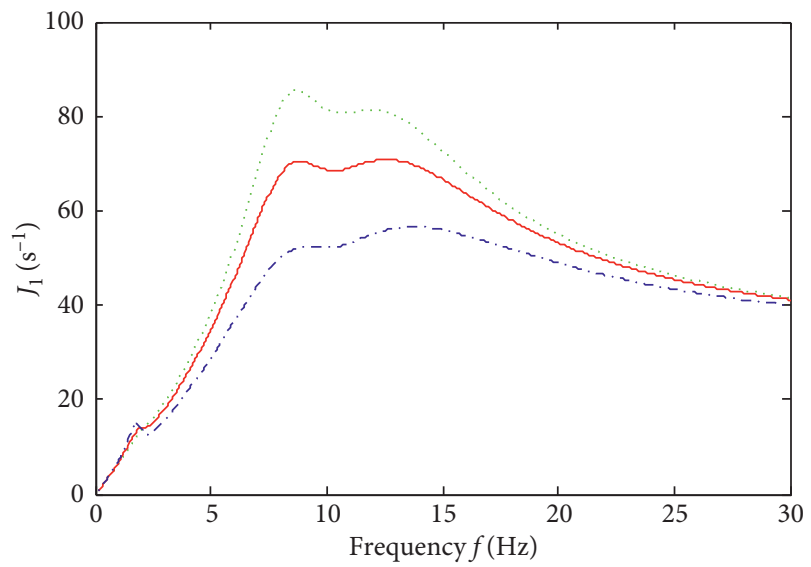

$$
\begin{aligned}
\ldots & r_{2}=1.5 \\
- & r_{2}=3.0 \\
-.- & r_{2}=6.0
\end{aligned}
$$

(a)

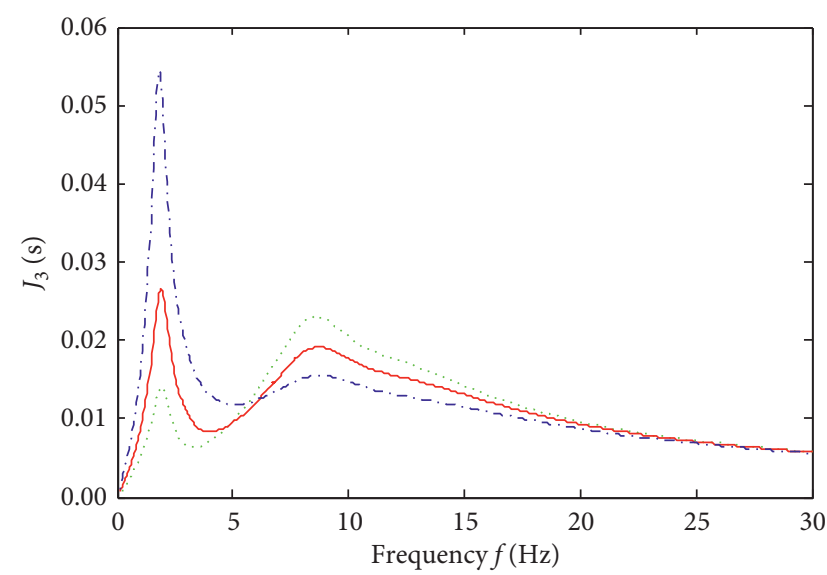

$r_{2}=1.5$

$-r_{2}=3.0$

...- $r_{2}=6.0$

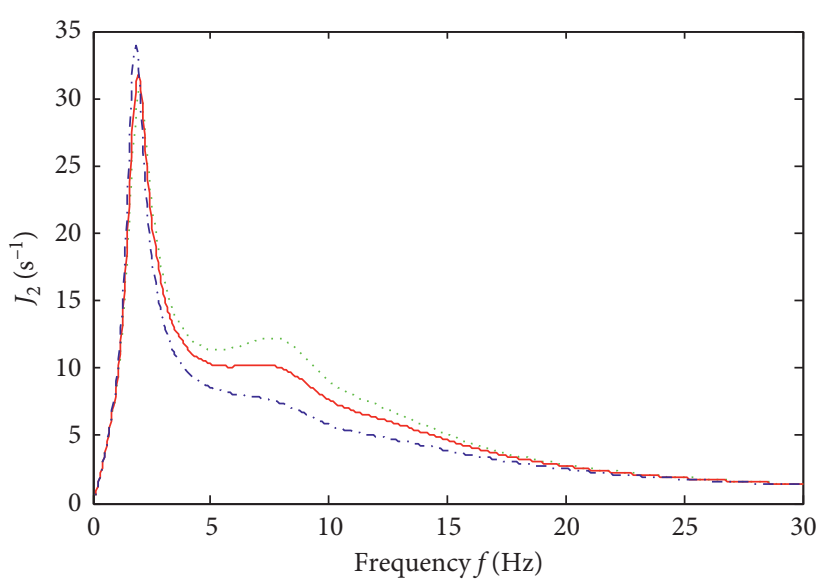

$$
\begin{array}{ll}
\ldots . . & r_{2}=1.5 \\
- & r_{2}=3.0 \\
\ldots- & r_{2}=6.0
\end{array}
$$

(b)

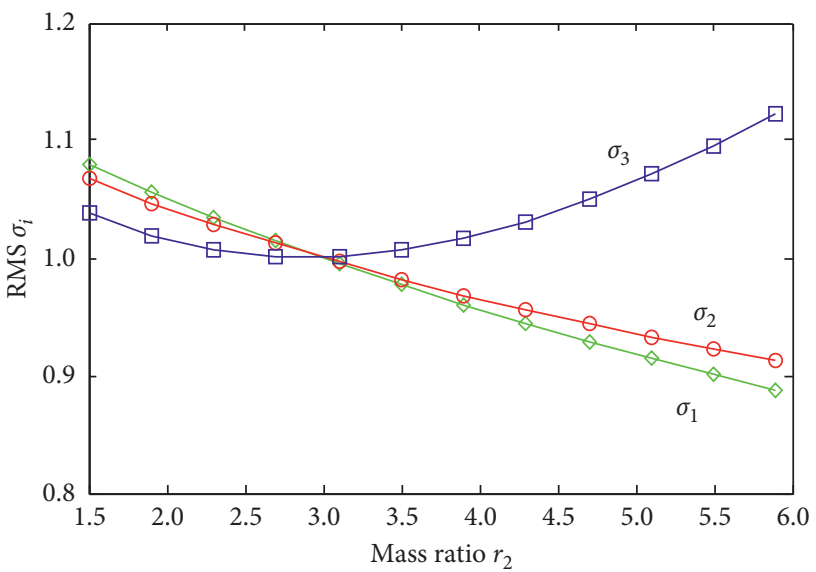

(d)

FIgURE 4: Influences of $r_{2}$ on $\ddot{z}_{1}, \ddot{z}_{2}$, and $f_{\mathrm{d}}$ : (a) the amplitude frequency characteristics $J_{1}$ at different values of $r_{2}$; (b) the amplitude frequency characteristics $J_{2}$ at different values of $r_{2}$; (c) the amplitude frequency characteristics $J_{3}$ at different values of $r_{2}$; (d) the RMS $\sigma_{1}$, $\sigma_{2}$, and $\sigma_{3}$ versus $r_{2}$. 


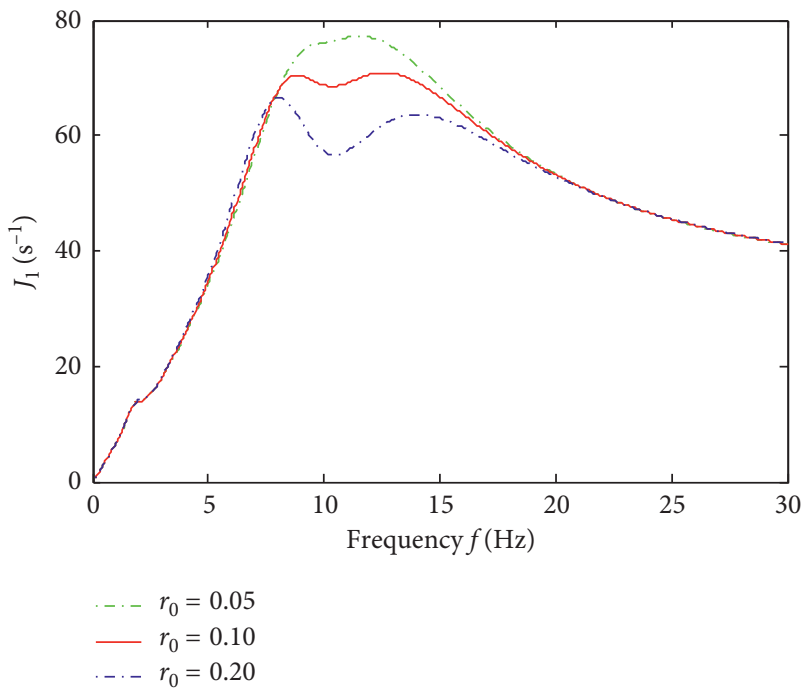

(a)

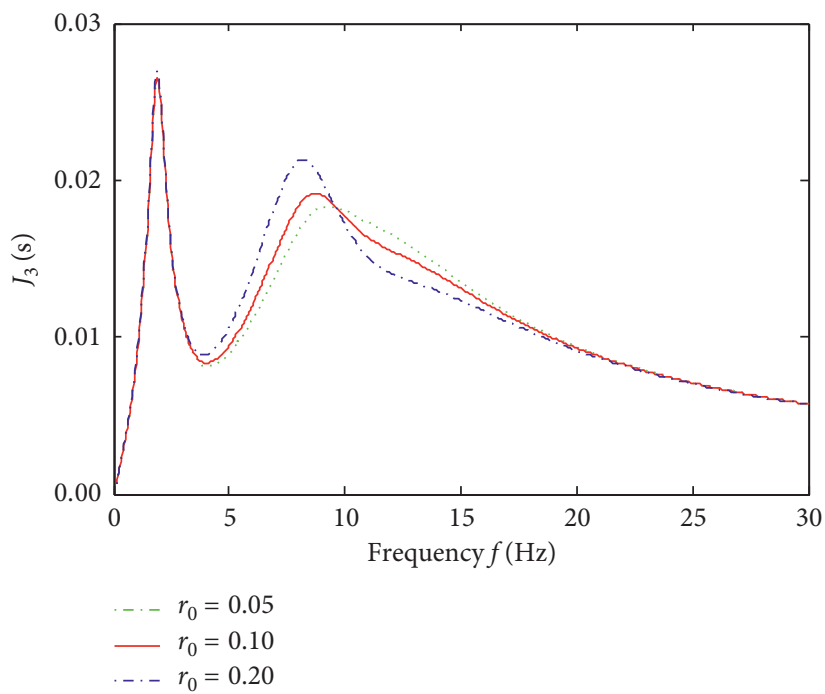

(c)

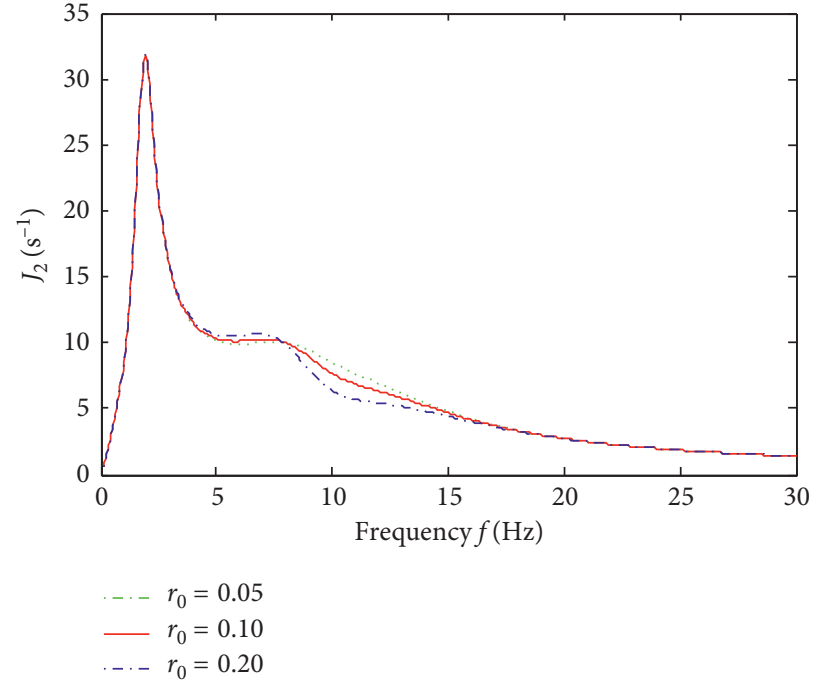

(b)

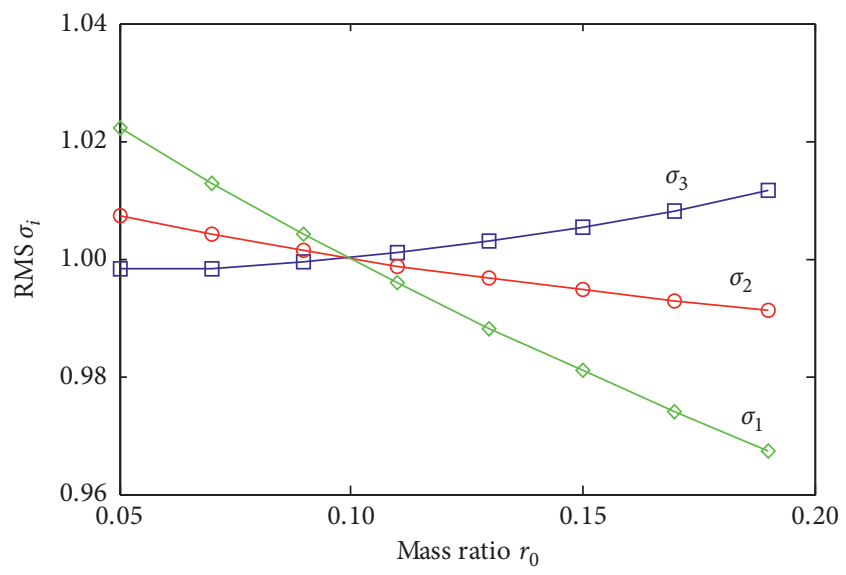

(d)

FIgURE 5: Influences of $r_{0}$ on $\ddot{z}_{1}, \ddot{z}_{2}$, and $f_{\mathrm{d}}$ : (a) the amplitude frequency characteristics $J_{1}$ at different values of $r_{0}$; (b) the amplitude frequency characteristics $J_{2}$ at different values of $r_{0}$; (c) the amplitude frequency characteristics $J_{3}$ at different values of $r_{0}$; (d) the RMS $\sigma_{1}$, $\sigma_{2}$, and $\sigma_{3}$ versus $r_{0}$.

\section{Vibration Characteristics Comparison}

In order to facilitate the vibration characteristics analysis of the convalescent-wheelchair system with DA, the convalescent-wheelchair system without DA is taken as a comparison object. Based on a typical commercially available wheelchair and the selection method of the DA [19], the values of the parameters for the convalescentwheelchair system is given in Table 1 [21]. The simulation condition is set as: $u=0.5 \sim 2.5 \mathrm{~m} / \mathrm{s}$, the $\mathrm{C}$ grade road with $G_{q}\left(n_{0}\right)=256 \times 10^{-6} \mathrm{~m}^{-3}$. For the numerical calculation, $\Delta f=0.1 \mathrm{~Hz}$, and the upper limit frequency is set as $30 \mathrm{~Hz}$. Based on the vibration models of the convalescentwheelchair system with DA and without DA, the values of $\sigma_{\ddot{z}_{2}}, \sigma_{\ddot{z}_{1}}, \sigma_{f_{\mathrm{d}}}, G_{\ddot{z}_{2}}, G_{\ddot{z}_{1}}$, and $G_{f_{\mathrm{d}}}$ were numerically calculated, respectively. The calculated results are shown in Figure 3.

Figures 3(a)-3(c) show that the DA can effectively reduce the wheelchair body RMS acceleration $\sigma_{\ddot{z}}$ and the convalescent RMS acceleration $\sigma_{\ddot{z}_{2}}$, and the TDD RMS $\sigma_{f_{\mathrm{d}}}$ is not improved obviously. Figure $3(\mathrm{~d})$ shows that the peak value of the wheelchair body acceleration PSD $G_{\ddot{z}_{1}}$ is decreased by $40.2 \%$ by using the DA. Figure 3(e) illustrates that the DA reduces the convalescent acceleration PSD $G_{\ddot{z}_{2}}$ around $10 \mathrm{~Hz}$ but causes a slight increase in the range of $4 \sim 7 \mathrm{~Hz}$. Figure 3(f) depicts that the DA makes the peak value of the TDD PSD $G_{f_{\mathrm{d}}}$ shift to the left. The comparison results show that the DA partly suppresses the vibration of the convalescent and the wheelchair body, especially in the resonance area of the wheelchair body. However, the 

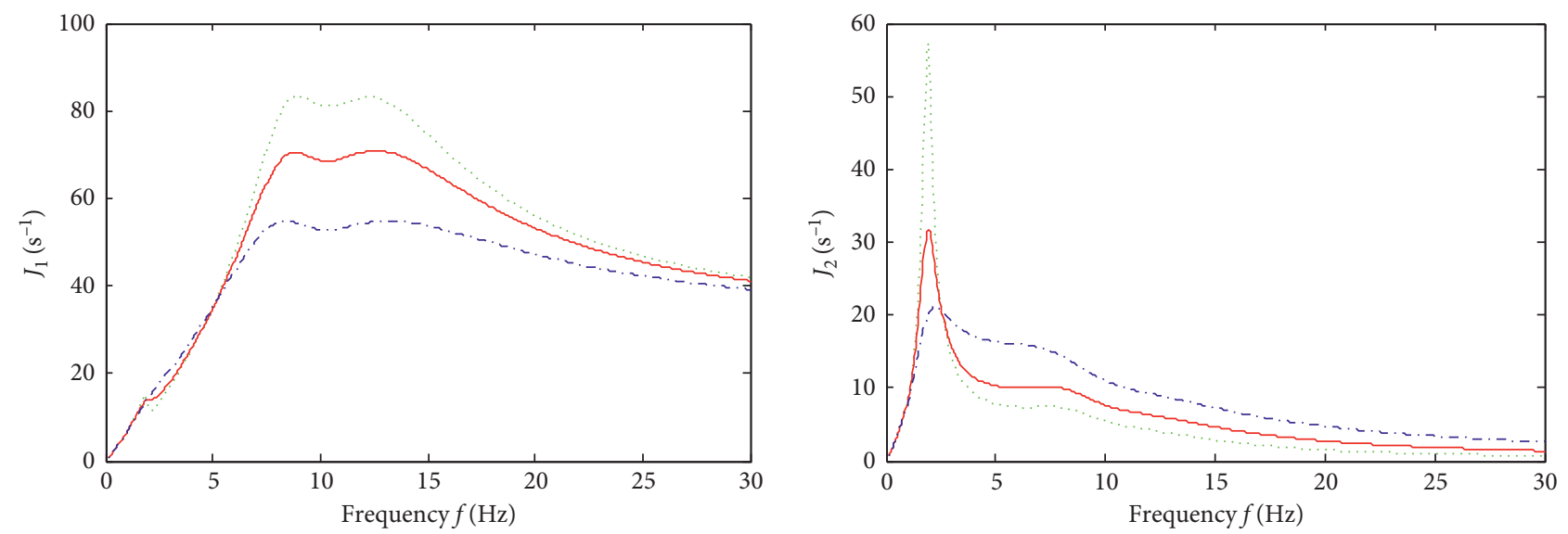

$$
\begin{aligned}
\xi_{2} & =0.125 \\
- & \xi_{2}=0.25 \\
\ldots \quad & \xi_{2}=0.50
\end{aligned}
$$

$$
\begin{aligned}
\ldots . . \quad \xi_{2} & =0.125 \\
\ldots & \xi_{2}=0.25 \\
\ldots . .-\xi_{2} & =0.50
\end{aligned}
$$

(a)

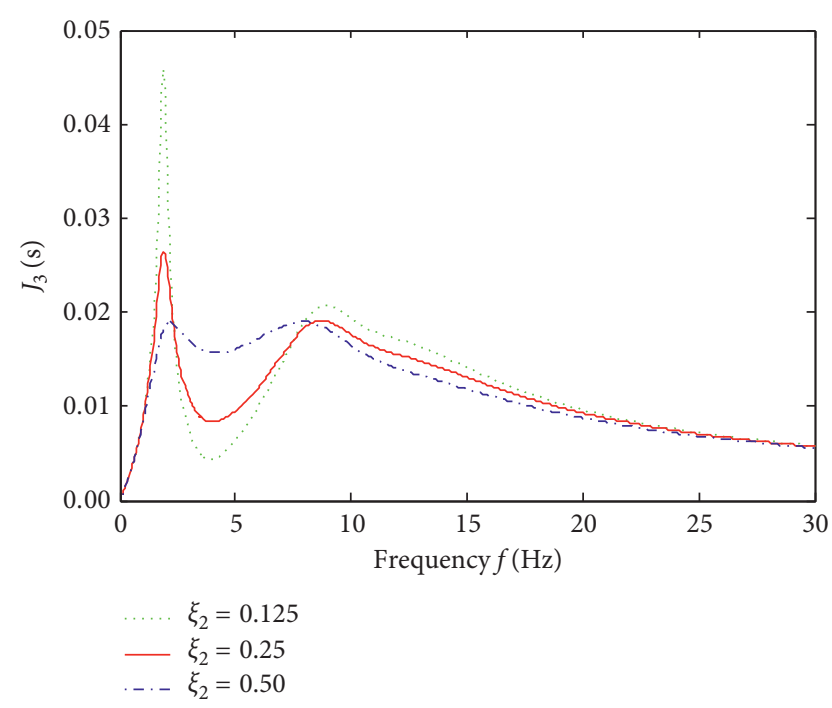

(b)

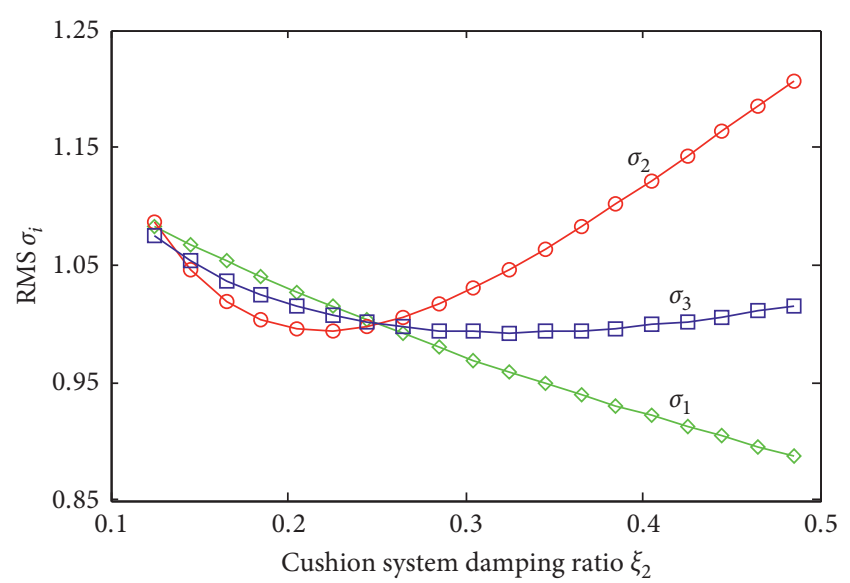

(c)

(d)

FIGURE 6: Influence of $\xi_{2}$ on $\ddot{z}_{1}, \ddot{z}_{2}$, and $f_{\mathrm{d}}$ : (a) the amplitude frequency characteristics $J_{1}$ at different values of $\xi_{2}$; (b) the amplitude frequency characteristics $J_{2}$ at different values of $\xi_{2}$; (c) the amplitude frequency characteristics $J_{3}$ at different values of $\xi_{2}$; (d) the RMS $\sigma_{1}$, $\sigma_{2}$, and $\sigma_{3}$ versus $\xi_{2}$.

DA does not successfully improve the tire contact behavior.

\section{Sensitivity Analysis of Vibration Responses to System Parameters}

In order to analyze the effects of $r_{2}, r_{0}, \xi_{1}, \xi_{2}, \xi_{0}, f_{0}, f_{1}$, and $f_{2}$ on $\sigma_{\ddot{z}_{2}}, \sigma_{\ddot{z}_{1}}$, and $\sigma_{f_{\mathrm{d}}}$, the simulation calculation was carried out based on the vibration model of the convalescentwheelchair system with DA. The simulation condition is set as $u=0.1 \mathrm{~m} / \mathrm{s}$, the $\mathrm{C}$ grade road with $G_{q}\left(n_{0}\right)=$ $256 \times 10^{-6} \mathrm{~m}^{-3}$. To represent the curve changes in the same diagram, the values of $\sigma_{\ddot{z}_{2}}, \sigma_{\ddot{z}_{1}}$, and $\sigma_{f_{\mathrm{d}}}$ are normalized on the basis of the baseline RMS values, respectively. The processing method is as follows: $\sigma_{2}=\sigma_{\ddot{z}_{2}} /\left|\sigma_{\ddot{z}_{2}}\right|_{\mathrm{b}}, \sigma_{1}=\sigma_{\ddot{z}_{1}} /\left|\sigma_{\ddot{z}_{1}}\right|_{\mathrm{b}}$, $\sigma_{3}=\sigma_{f_{\mathrm{d}}} /\left|\sigma_{f_{\mathrm{d}}}\right|_{\mathrm{b}}$, where the subscript "b" represents "baseline." In addition, to observe the performance changes in the local frequency band, the amplitude frequency characteristics functions $J_{1}, J_{2}$, and $J_{3}$ are also calculated.

When the influence of one of the eight parameters is analyzed, the baseline value is increased by $100 \%$ or decreased by $50 \%$, and the other parameters are fixed. The values of the system parameters for the analysis are given in Table 2 [21]. The baseline values are converted from Table 1. 

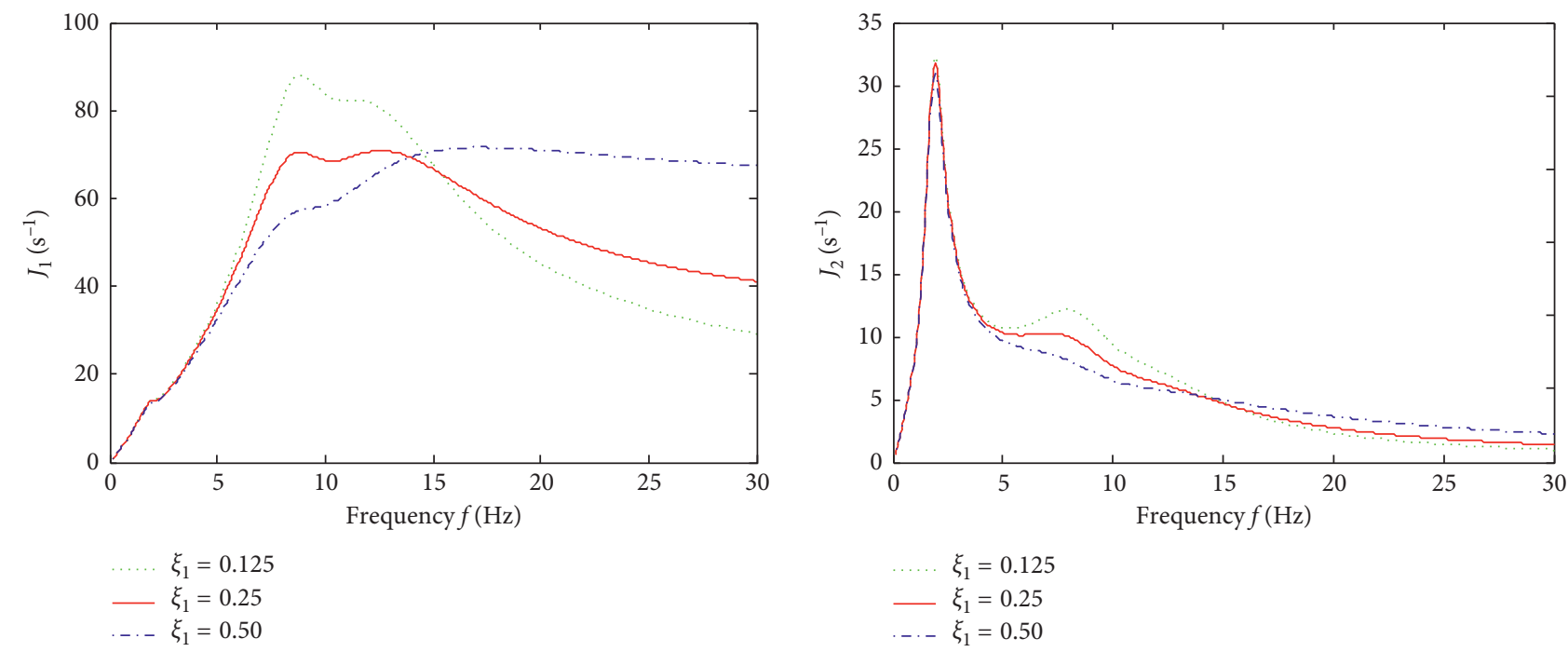

(a)

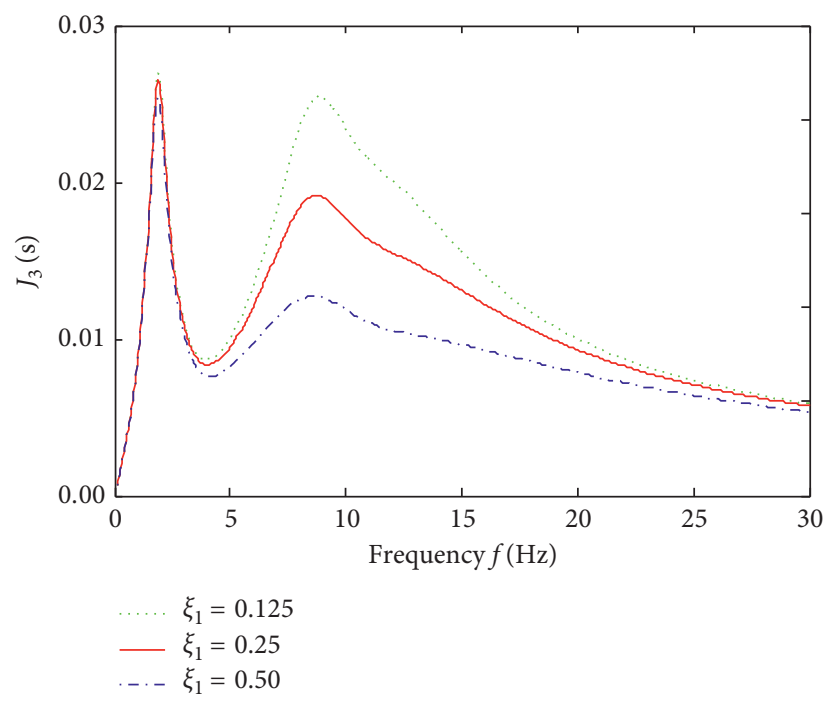

(b)

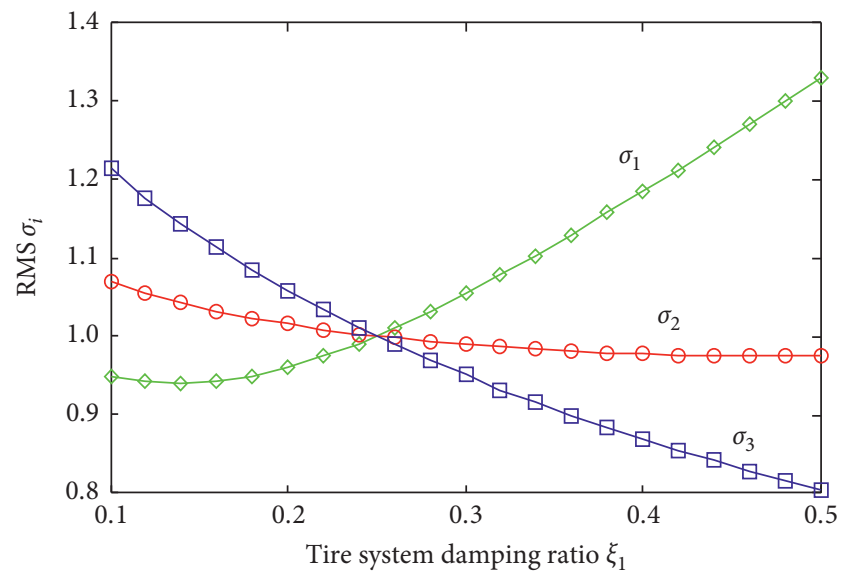

(c)

(d)

Figure 7: Influences of $\xi_{1}$ on $\ddot{z}_{1}, \ddot{z}_{2}$, and $f_{\mathrm{d}}$ : (a) the amplitude frequency characteristics $J_{1}$ at different values of $\xi_{1}$; (b) the amplitude frequency characteristics $J_{2}$ at different values of $\xi_{1}$; (c) the amplitude frequency characteristics $J_{3}$ at different values of $\xi_{1}$; (d) the RMS $\sigma_{1}$, $\sigma_{2}$, and $\sigma_{3}$ versus $\xi_{1}$.

\subsection{The Influences of the Mass Ratios}

6.1.1. The Influences of the Mass Ratio $r_{2}$. Figure 4 depicts the influences of $r_{2}$ on $\ddot{z}_{1}, \ddot{z}_{2}$, and $f_{\mathrm{d}}$. From Figure $4(\mathrm{a})$, it can be seen that increasing $r_{2}$ can almost reduce the amplitude of $J_{1}$ in the whole frequency domain. Therefore, increasing $r_{2}$ is conducive to reducing the wheelchair body acceleration. Figure 4(b) illustrates that increasing $r_{2}$ can reduce the amplitude of $J_{2}$ in the range of $5 \sim 15 \mathrm{~Hz}$, but it causes the amplitude to increase around the low frequency resonance peak. From Figure 4(c), it can be seen that with the increase of $r_{2}$, the low frequency resonance peak of $J_{3}$ increases sharply, while the high frequency formant decreases. Therefore, too large $r_{2}$ is not conducive to improving the tire grounding safety. As shown in Figure 4(d), $\sigma_{1}$ and $\sigma_{2}$ are in inverse proportion to $r_{2}$, and $\sigma_{3}$ has a minimum value for $r_{2}$ in the range of $2.5 \sim 3.5$. Thus, the increase of $r_{2}$ is mainly to reduce the values of $\sigma_{1}$ and $\sigma_{2}$.

6.1.2. The Influences of the Mass Ratio $r_{0}$. Figure 5 shows the influences of $r_{0}$ on $\ddot{z}_{1}, \ddot{z}_{2}$, and $f_{\mathrm{d}}$. From Figure $5(\mathrm{a})$, it can be seen that increasing $r_{0}$ can effectively reduce the peak value of $J_{1}$. Figure $5(\mathrm{~b})$ shows that increasing $r_{0}$ can reduce the amplitude of $J_{2}$ from $7 \mathrm{~Hz}$ to $15 \mathrm{~Hz}$, but the amplitude in the range of $4 \sim 7 \mathrm{~Hz}$ increases slightly. From Figure 5(c), it can be seen that the larger the value of $r_{0}$ is, the higher the formant of $J_{3}$ is. As shown in Figure 5(d), $\sigma_{1}$ and $\sigma_{2}$ are in inverse proportion to $r_{0}, \sigma_{3}$ is proportional to $r_{0}$, and $\sigma_{1}$ is most sensitive to the change of $r_{0}$. Thus, the use of the DA with the larger mass helps to reduce the vibration of the convalescent and the wheelchair. 

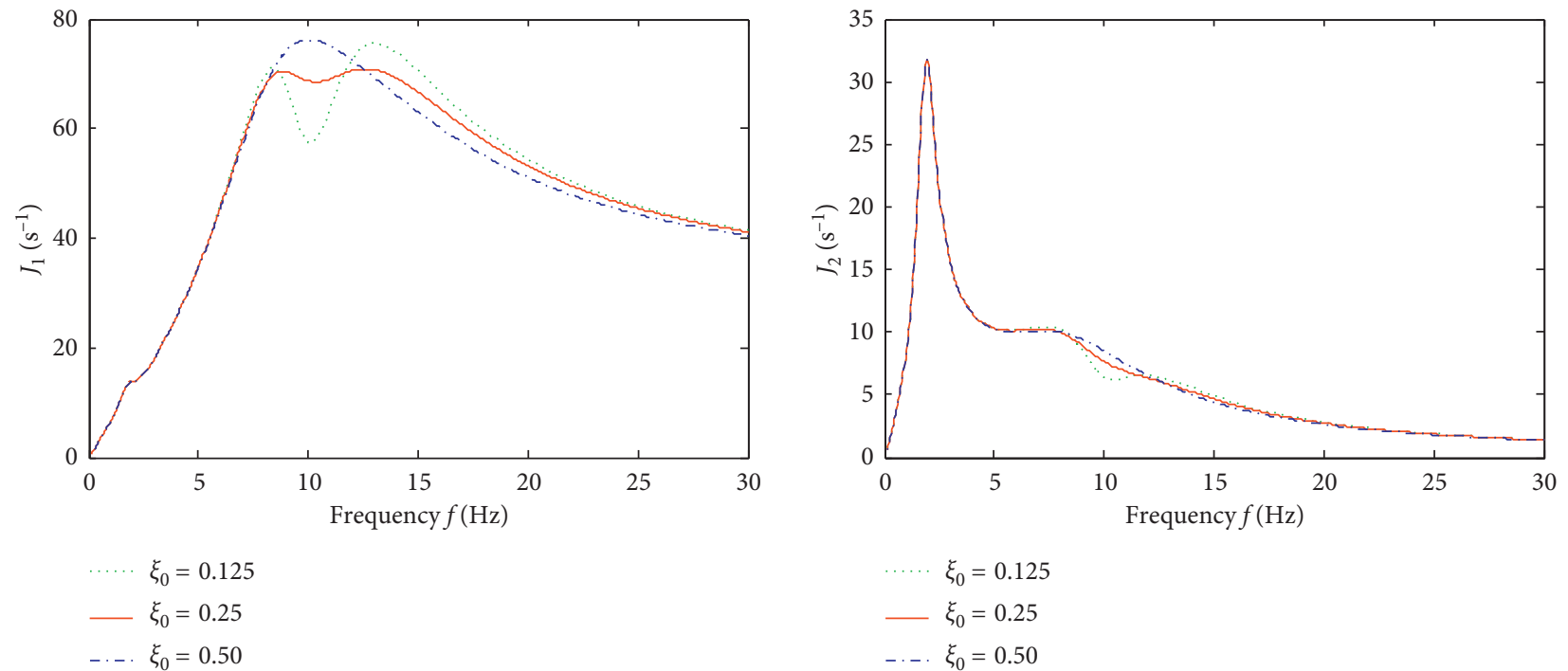

(a)

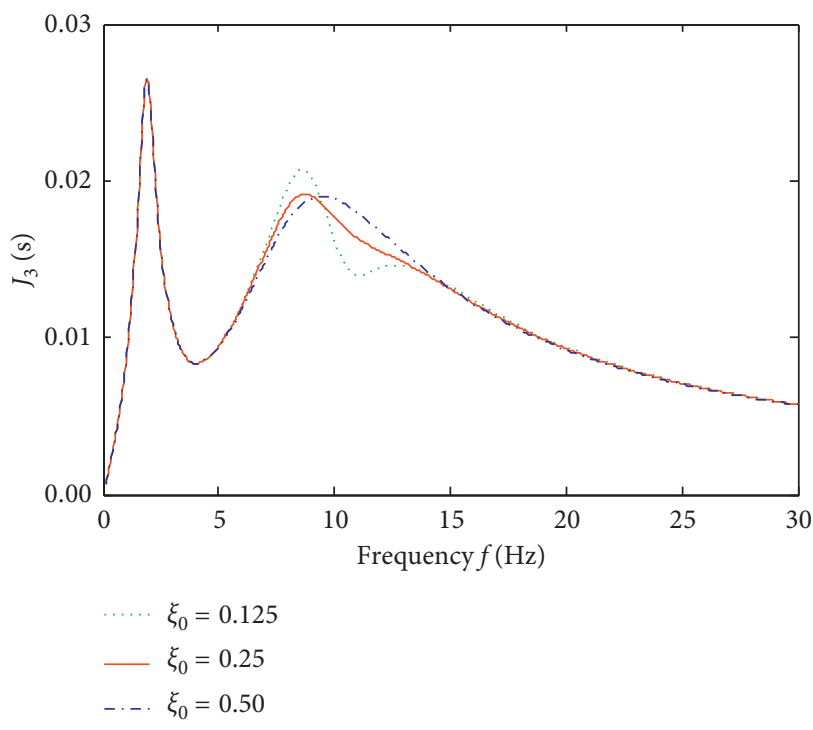

(b)

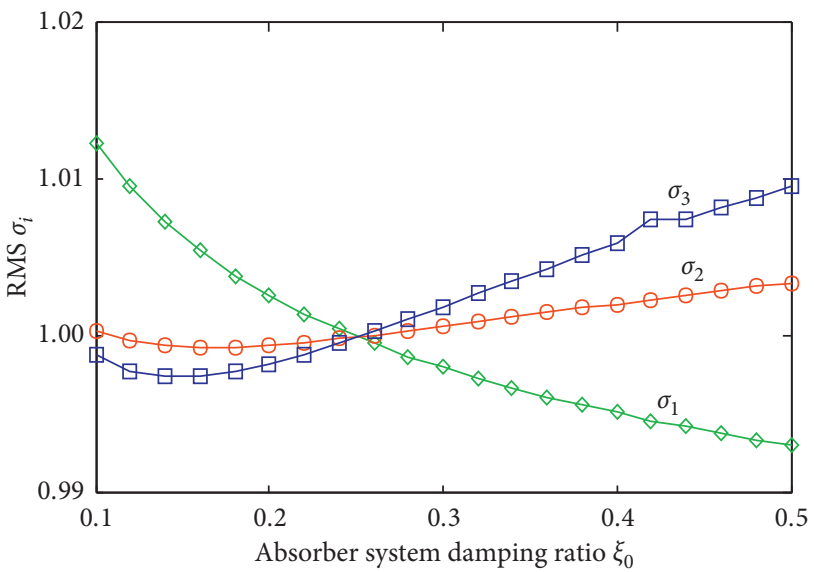

(c)

(d)

FiguRE 8: Influences of $\xi_{0}$ on $\ddot{z}_{1}, \ddot{z}_{2}$, and $f_{\mathrm{d}}$ : (a) the amplitude frequency characteristics $J_{1}$ at different values of $\xi_{0}$; (b) the amplitude frequency characteristics $J_{2}$ at different values of $\xi_{0}$; (c) the amplitude frequency characteristics $J_{3}$ at different values of $\xi_{0}$; (d) the RMS $\sigma_{1}$, $\sigma_{2}$, and $\sigma_{3}$ versus $\xi_{0}$.

\subsection{The Influences of the Damping Ratios}

6.2.1. The Influences of the Damping Ratio $\xi_{2}$. Figure 6 depicts the influences of $\xi_{2}$ on $\ddot{z}_{1}, \ddot{z}_{2}$, and $f_{\mathrm{d}}$. As shown in Figure $6(\mathrm{a})$, increasing $\xi_{2}$ can help to reduce the peak value of $J_{1}$. From Figure $6(\mathrm{~b})$, it can be seen that increasing $\xi_{2}$ can effectively reduce the low frequency resonance peak of $J_{2}$, but the amplitude after $3.0 \mathrm{~Hz}$ increases. Figure 6(c) shows that the larger the value of $\xi_{2}$ is, the smaller the peak values of $J_{3}$ are, but the amplitude between the two formants increases obviously. Figure 5(d) illustrates that $\sigma_{1}$ is almost in inverse proportional to $\xi_{2}$, there is a minimum value of $\sigma_{2}$ for the value of $\xi_{2}$ between $0.15 \sim 0.25$, and $\sigma_{3}$ has a minimum value when the value of $\xi_{2}$ belongs to $0.15 \sim 0.25$. The ride comfort requires that the cushion system has the smaller value of $\xi_{2}$, however, the tire grounding safety and the operational reliability need the larger value of $\xi_{2}$. Thus, taking the smaller value of $\xi_{2}$, about 0.2 , is helpful to improve comfort.

6.2.2. The Influences of the Damping Ratio $\xi_{1}$. Figure 7 depicts the influences of $\xi_{1}$ on $\ddot{z}_{1}, \ddot{z}_{2}$, and $f_{\mathrm{d}}$. From Figure $7(\mathrm{a})$, it can be seen that increasing $\xi_{1}$ can effectively suppress the peak value of $J_{1}$, but the amplitude after $15 \mathrm{~Hz}$ increases. As shown in Figure $7(\mathrm{~b})$, the increase of $\xi_{1}$ can effectively suppress the low frequency and the high frequency resonance peak values of $J_{2}$, and the suppression effectiveness around the high frequency resonance peak is more obvious. However, the amplitude after $15 \mathrm{~Hz}$ 


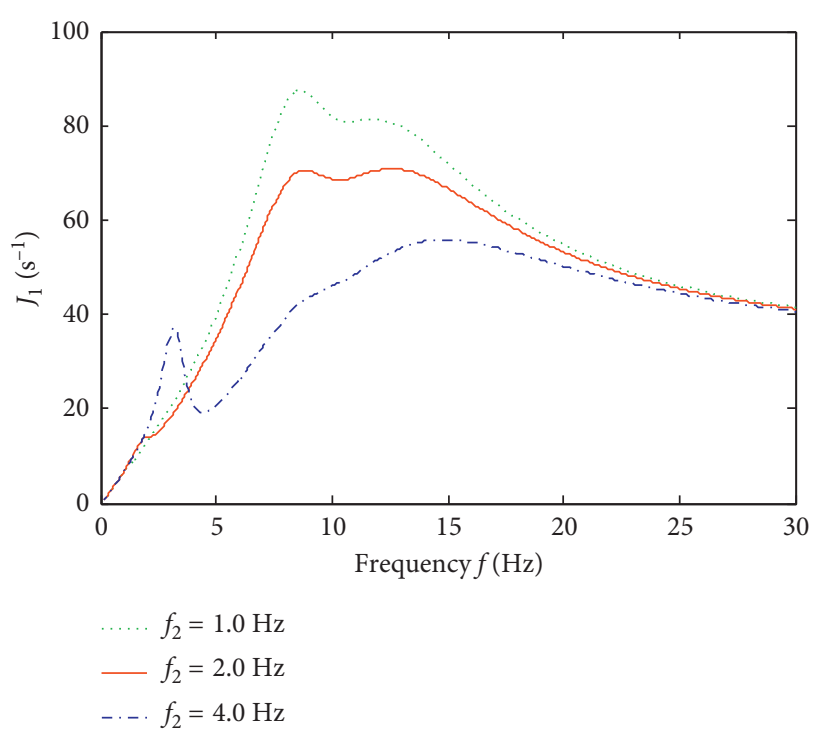

(a)

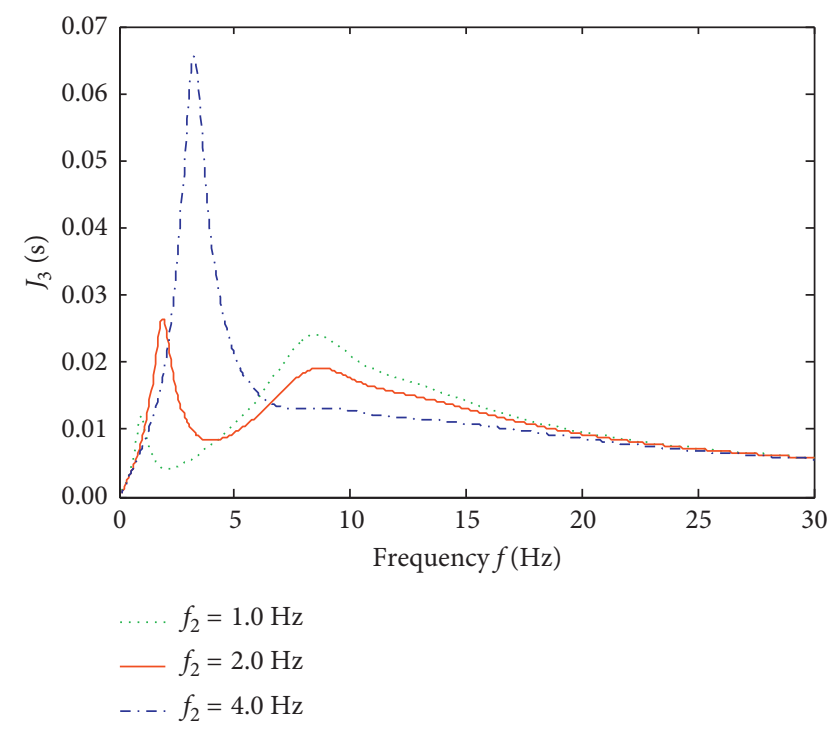

(c)

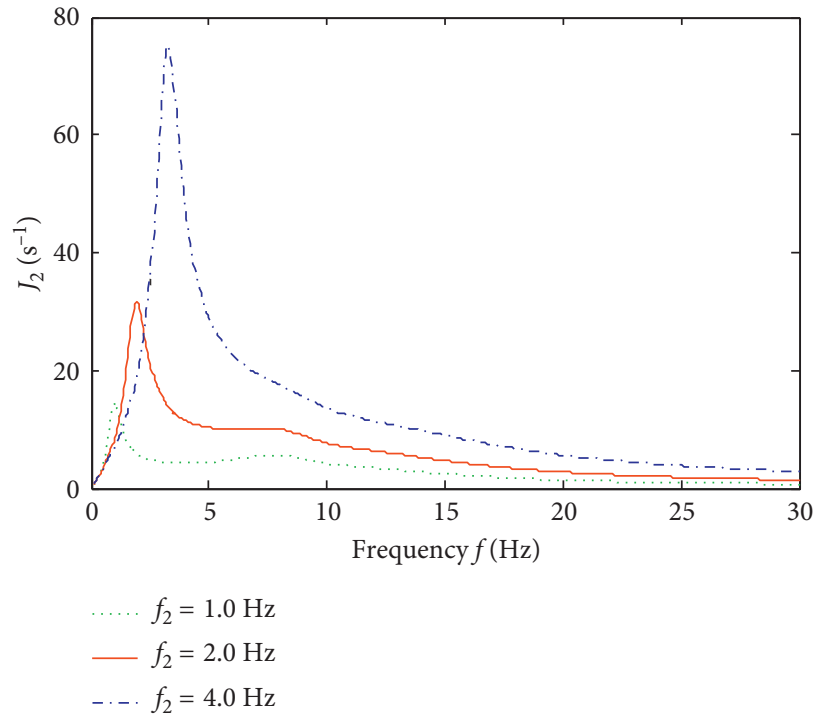

(b)

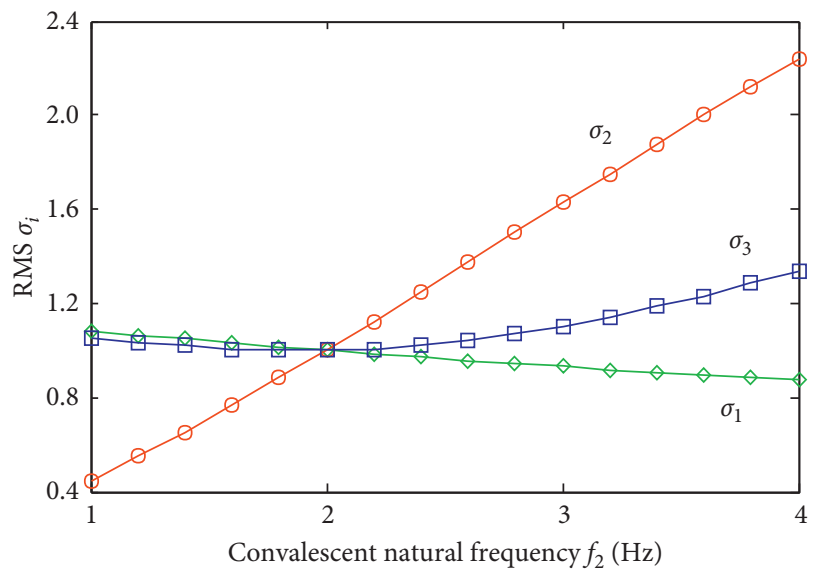

(d)

FIGURE 9: Influences of $f_{2}$ on $\ddot{z}_{1}, \ddot{z}_{2}$, and $f_{\mathrm{d}}$ : (a) the amplitude frequency characteristics $J_{1}$ at different values of $f_{2}$; (b) the amplitude frequency characteristics $J_{2}$ at different values of $f_{2}$; (c) the amplitude frequency characteristics $J_{3}$ at different values of $f_{2}$; (d) the RMS $\sigma_{1}$, $\sigma_{2}$, and $\sigma_{3}$ versus $f_{2}$.

also increases. Figure 7(c) clearly shows that increasing $\xi_{1}$ can almost reduce the amplitude of $J_{3}$ in the full frequency domain. From Figure $7(\mathrm{~d})$, it can be seen that both $\sigma_{2}$ and $\sigma_{3}$ are approximately in inverse proportion to $\xi_{1}$, and when the value of $\xi_{1}$ belongs to $0.1 \sim 0.2$, a minimum value of $\sigma_{1}$ exists. Thus, the ride comfort and the tire grounding safety require that the tire system has the larger value of $\xi_{1}$, however, the operational reliability needs the smaller value of $\xi_{1}$.

6.2.3. The Influences of the Damping Ratio $\xi_{0}$. Figure 8 depicts the influences of $\xi_{0}$ on $\ddot{z}_{1}, \ddot{z}_{2}$, and $f_{\mathrm{d}}$. According to Figure $8(\mathrm{a})$, the amplitude of $J_{1}$ in the region of $8 \sim 13 \mathrm{~Hz}$ increases with the increase of $\xi_{0}$, and the amplitude decreases after $13 \mathrm{~Hz}$. Figure 8(b) shows that increasing $\xi_{0}$ can effectively suppress the high frequency resonance peak value of $J_{2}$. According to Figure 8(c), $\xi_{0}$ has obvious effect on the high frequency resonance peak value of $J_{3}$. From Figure $8(\mathrm{~d})$, it can be seen that, with the increase of $\xi_{0}$, the value of $\sigma_{1}$ decreases. When $\xi_{0}$ belongs to $0.1 \sim 0.2, \sigma_{3}$ has a minimum value, and when $\xi_{0}$ belongs to $0.15 \sim 0.25, \sigma_{2}$ has a minimum value. When the value of $\xi_{0}$ equals 0.2 , the DA system can well give consideration to the three indexes: the ride comfort, the tire grounding safety, and the operational reliability.

\subsection{The Influences of the Natural Frequencies}

6.3.1. The Influences of the Natural Frequency $f_{2}$. Figure 9 depicts the influences of $f_{2}$ on $\ddot{z}_{1}, \ddot{z}_{2}$, and $f_{\mathrm{d}}$. From Figure 9(a), it can be seen that increasing $f_{2}$ is beneficial to 


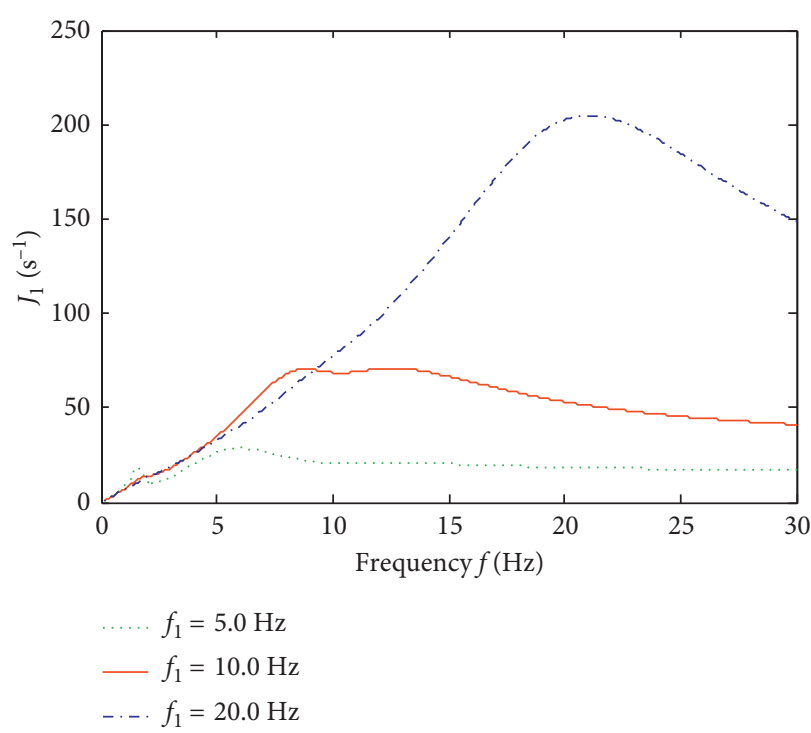

(a)

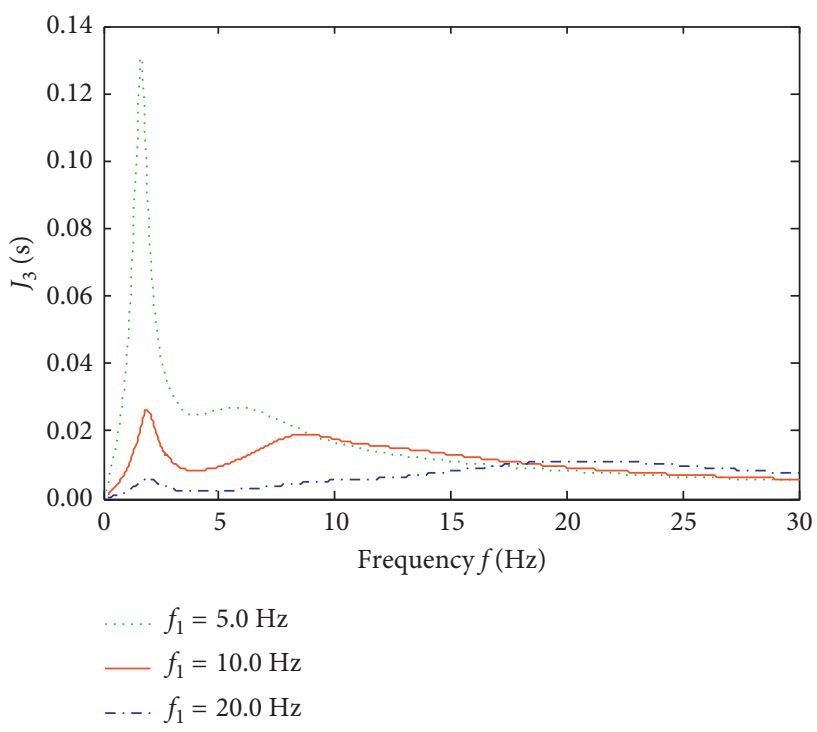

(c)

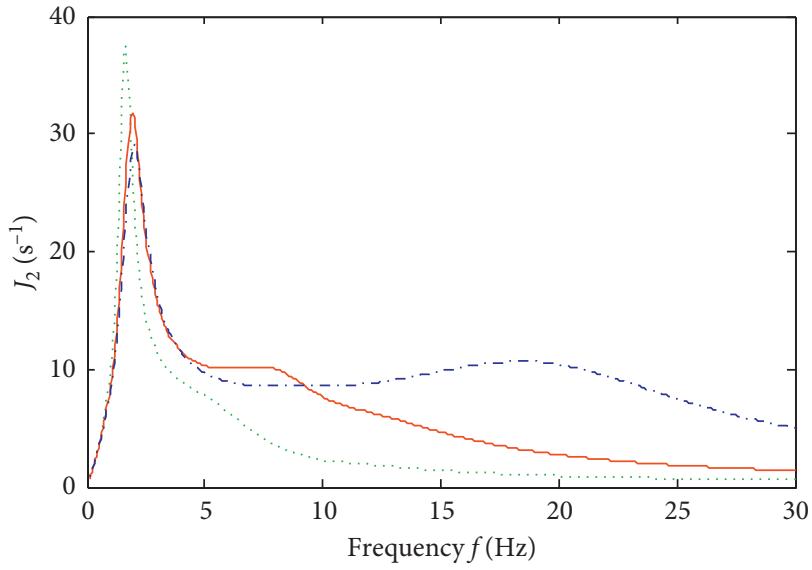

$\begin{aligned} \ldots . . f_{1} & =5.0 \mathrm{~Hz} \\ -f_{1} & =10.0 \mathrm{~Hz} \\ \ldots .-f_{1} & =20.0 \mathrm{~Hz}\end{aligned}$

(b)

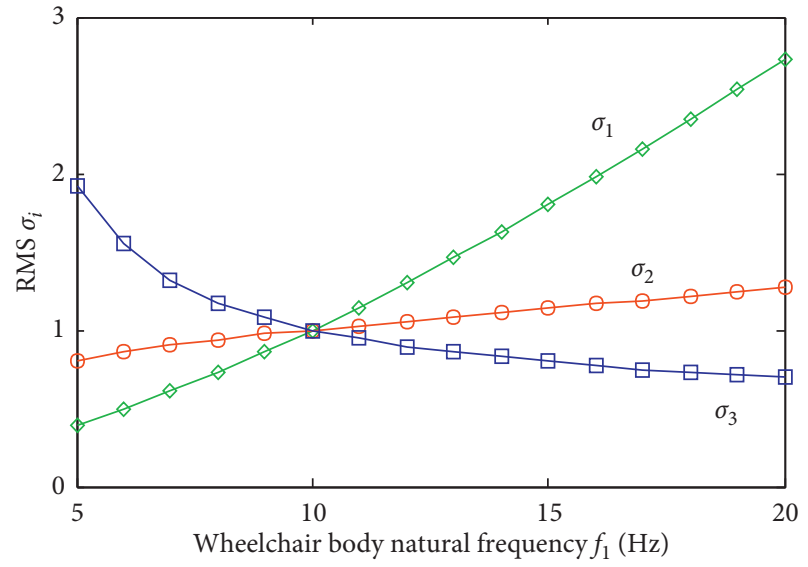

(d)

FIGURE 10: Influences of $f_{1}$ on $\ddot{z}_{1}, \ddot{z}_{2}$, and $f_{\mathrm{d}}$ : (a) the amplitude frequency characteristics $J_{1}$ at different values of $f_{1}$; (b) the amplitude frequency characteristics $J_{2}$ at different values of $f_{1}$; (c) the amplitude frequency characteristics $J_{3}$ at different values of $f_{1}$; (d) the RMS $\sigma_{1}$, $\sigma_{2}$, and $\sigma_{3}$ versus $f_{1}$.

suppress the resonance peak value of $J_{1}$. Figures 9 (b) and 9(c) show that increasing $f_{2}$ makes the low frequency formants of $J_{2}$ and $J_{3}$ increase sharply and shift rightward. As shown in Figure $9(\mathrm{~d}), \sigma_{1}$ is almost in inverse proportion to $f_{2}, \sigma_{2}$ is proportional to $f_{2}$ and $\sigma_{2}$ is most sensitive to the change of $f_{2}$, and when $f_{2}$ belongs to $1.5 \sim 2.5 \mathrm{~Hz}, \sigma_{3}$ has a minimum value. Therefore, adopting a soft cushion to reduce $f_{2}$ is an important measure to improve ride comfort.

6.3.2. The Influences of the Natural Frequency $f_{1}$. Figure 10 depicts the influences of $f_{1}$ on $\ddot{z}_{1}, \ddot{z}_{2}$, and $f_{\mathrm{d}}$. Figure 10(a) shows that with the increase of $f_{1}$, the resonance peak value of $J_{1}$ increases sharply and moves to the right. Figure 10(b) illustrates that increasing $f_{1}$ is beneficial to reduce the peak value of the low frequency resonance peak value of $J_{2}$, but the amplitude after $10 \mathrm{~Hz}$ increases significantly. Figure $10(\mathrm{c})$ shows that the increase of $f_{1}$ is conducive to significantly reducing the amplitude of $J_{3}$ from $0 \mathrm{~Hz}$ to $18 \mathrm{~Hz}$, although the amplitude increases slightly after $18 \mathrm{~Hz}$. Figure $10(\mathrm{~d})$ shows that $\sigma_{1}$ and $\sigma_{2}$ are proportional to $f_{1}$, and $\sigma_{3}$ nonlinearly decreases as $f_{1}$ increases. Thus, the adoption of soft tires is conducive to improving ride comfort and operational reliability.

6.3.3. The Influences of the Natural Frequency $f_{0}$. Figure 11 depicts the influences of $f_{0}$ on $\ddot{z}_{1}, \ddot{z}_{2}$, and $f_{\mathrm{d}}$. Figure 11(a) shows that the increase of $f_{0}$ has little effect on $J_{1}$ in the low frequency region $0 \sim 5 \mathrm{~Hz}$ and the high 

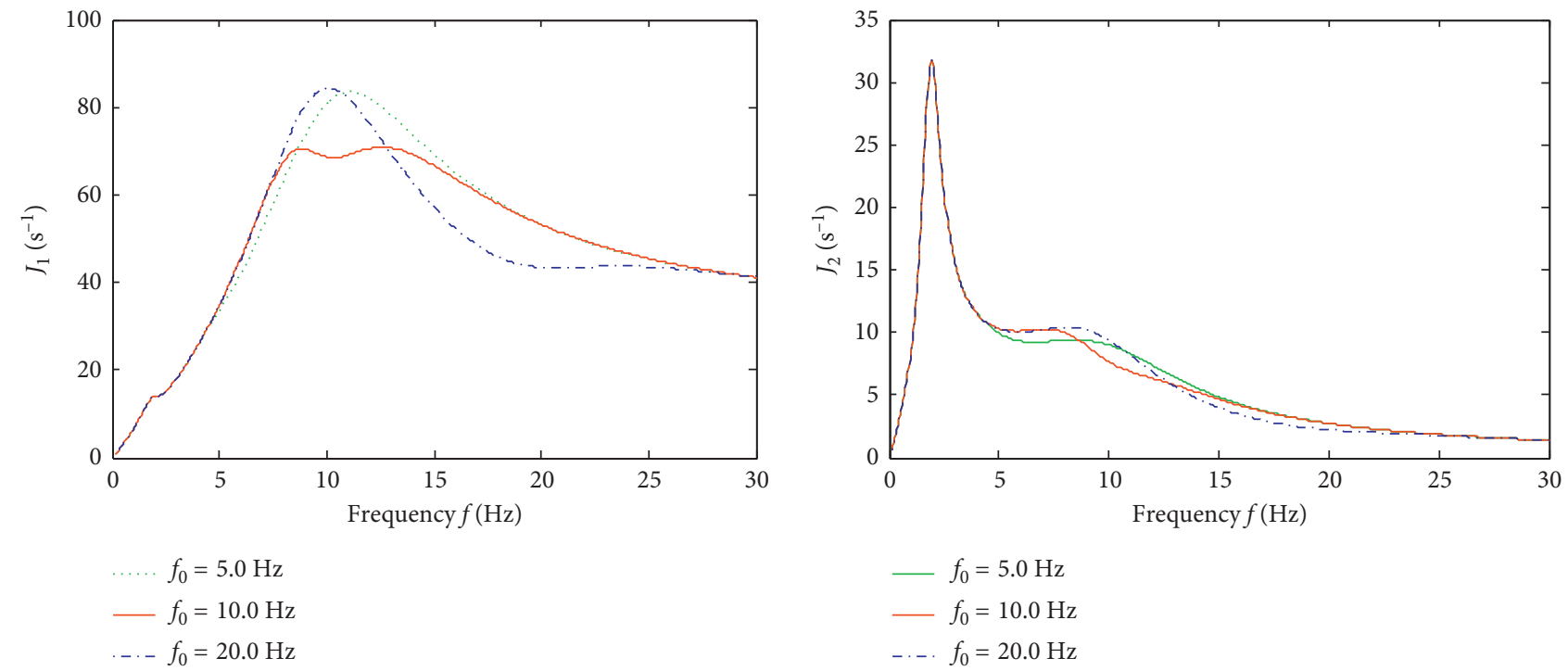

(a)
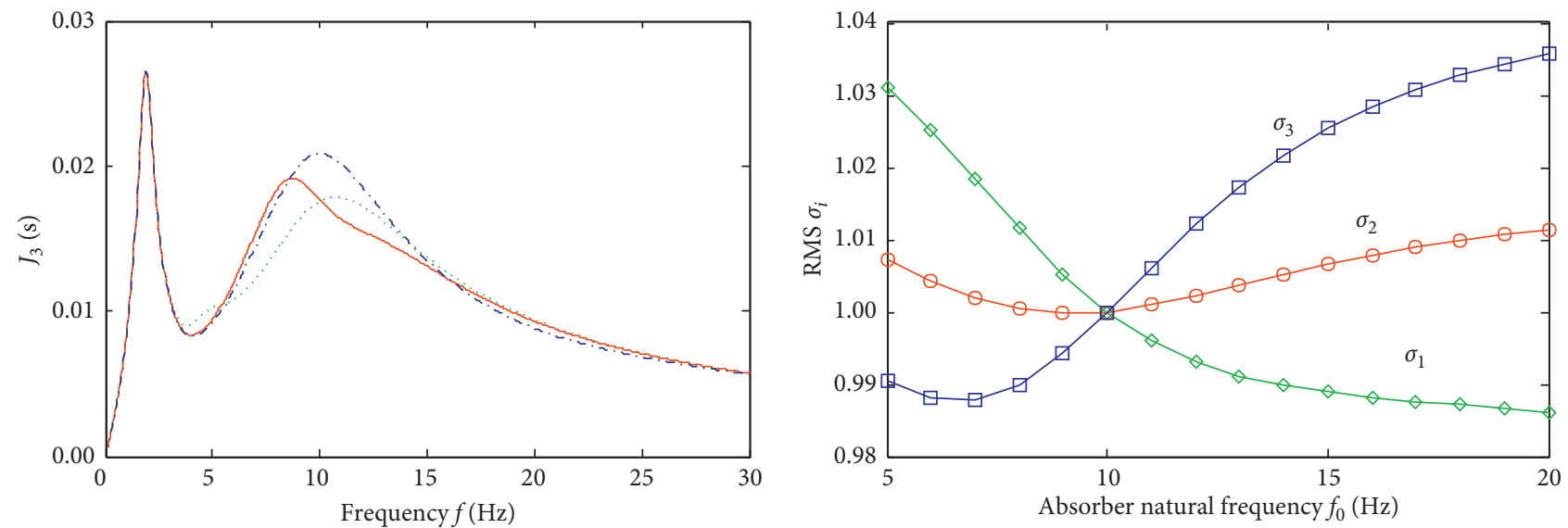

$$
\begin{aligned}
\ldots . . f_{0} & =5.0 \mathrm{~Hz} \\
-f_{0} & =10.0 \mathrm{~Hz} \\
--f_{0} & =20.0 \mathrm{~Hz}
\end{aligned}
$$

(c)

(d)

Figure 11: Influences of $f_{0}$ on $\ddot{z}_{1}, \ddot{z}_{2}$, and $f_{\mathrm{d}}$ : (a) the amplitude frequency characteristics $J_{1}$ at different values of $f_{0}$; (b) the amplitude frequency characteristics $J_{2}$ at different values of $f_{0}$; (c) the amplitude frequency characteristics $J_{3}$ at different values of $f_{0}$; (d) the RMS $\sigma_{1}$, $\sigma_{2}$, and $\sigma_{3}$ versus $f_{0}$.

TABLE 3: The sensitivity values of each response.

\begin{tabular}{lccc}
\hline System parameter & $s_{\ddot{z}_{1}}$ & $s_{\ddot{z}_{2}}$ & $s_{f_{d}}$ \\
\hline$r_{2}$ & 0.362 & 0.324 & 0.330 \\
$r_{0}$ & 0.198 & 0.107 & 0.099 \\
$\xi_{2}$ & 0.367 & 0.460 & 0.269 \\
$\xi_{1}$ & 0.498 & 0.242 & 0.505 \\
$\xi_{0}$ & 0.110 & 0.057 & 0.092 \\
$f_{2}$ & 0.376 & 1.095 & 0.511 \\
$f_{1}$ & 1.247 & 0.560 & 0.912 \\
$f_{0}$ & 0.173 & 0.113 & 0.184 \\
\hline
\end{tabular}

frequency $25 \sim 30 \mathrm{~Hz}$. In the mid frequency region $5 \sim 25 \mathrm{~Hz}$, increasing $f_{0}$ is beneficial to the amplitude reduction of $J_{1}$. Figure 11(b) shows that $f_{0}$ only obviously affects the amplitude of $J_{1}$ from $5 \mathrm{~Hz}$ to $20 \mathrm{~Hz}$. Figure 11 (c) clearly states that $f_{0}$ has a great influence on the high frequency resonance peak value of $J_{3}$. Figure $11(\mathrm{~d})$ illustrates that $\sigma_{1}$ nonlinearly decreases as $f_{0}$ increases, when the value of $f_{0}$ belongs to $8 \sim 12, \sigma_{2}$ has a minimum value, and when the value of $f_{0}$ belongs to $6 \sim 8, \sigma_{3}$ has a minimum value. Thus, when $f_{0}$ approximately equals to $f_{1}$, the DA system can well give consideration to the comprehensive isolation performance.

6.4. Sensitivity Calculation. Based on the influence trends analysis of the system parameters on the RMS responses in Sections 6.1 to 6.3 , sensitivity calculation was carried out by 


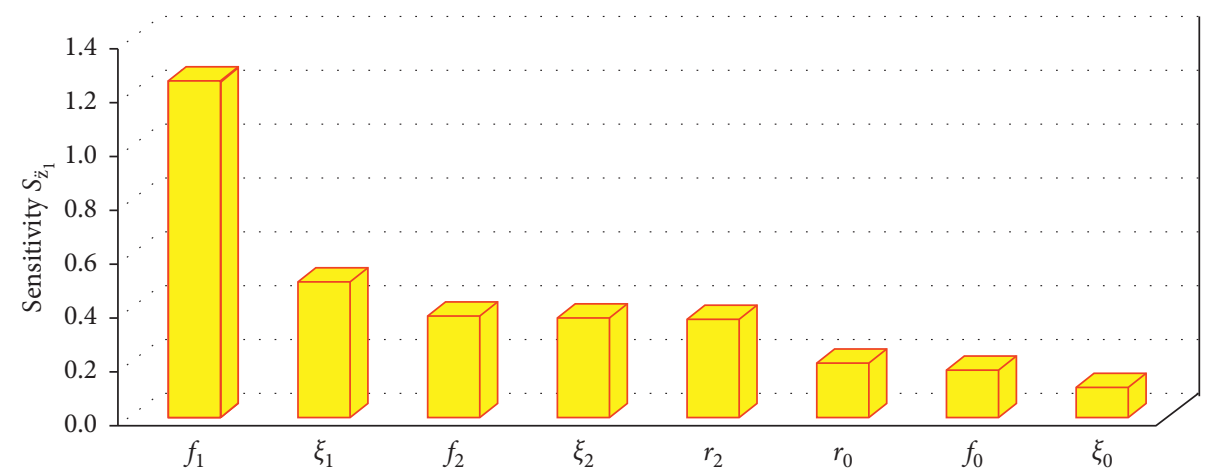

(a)

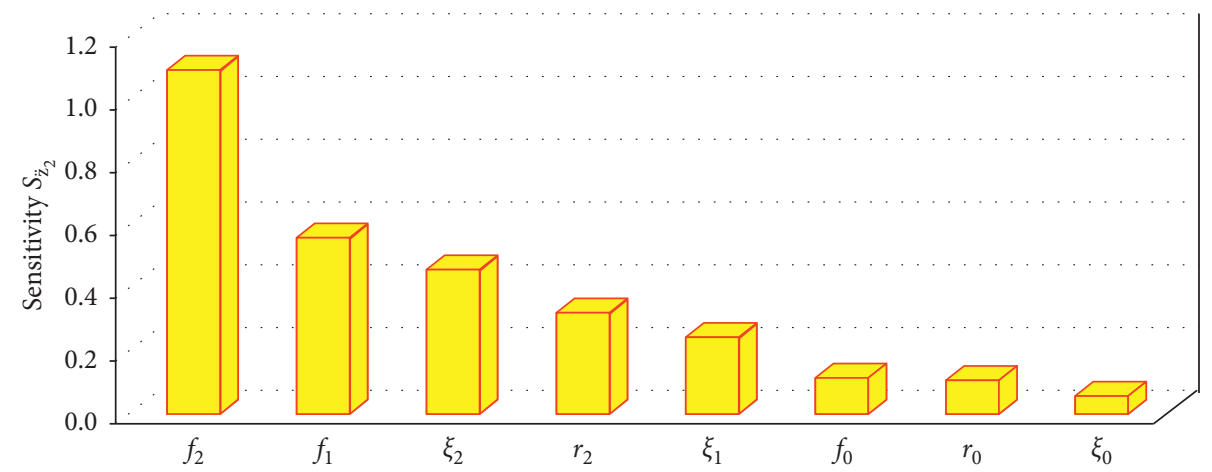

(b)

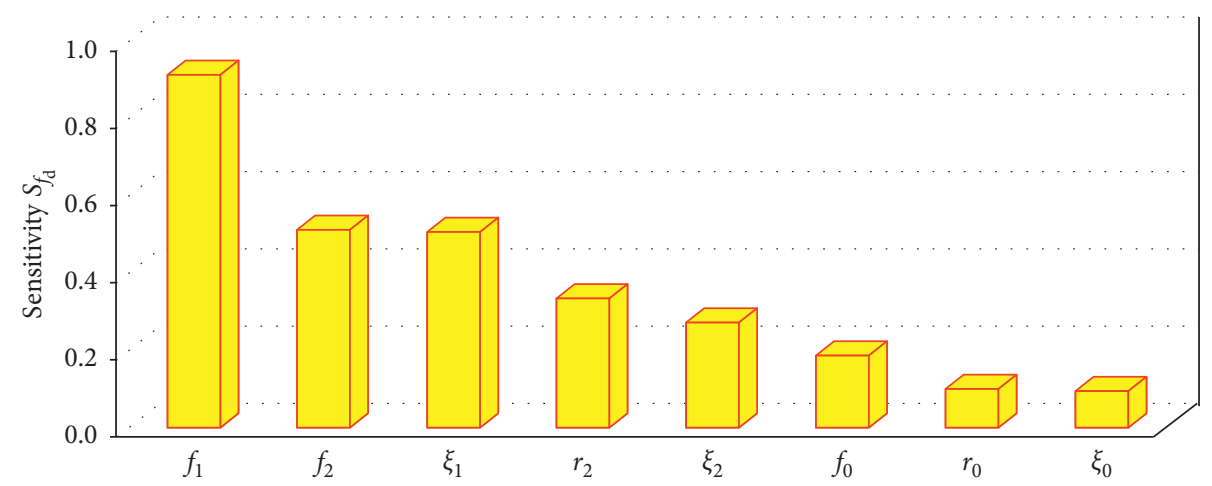

(c)

FIGURE 12: The vibration response sensitivity: (a) the wheelchair body RMS acceleration sensitivity $s_{\ddot{z}}$; (b) the convalescent RMS acceleration sensitivity $s_{\ddot{z}} ;(\mathrm{c})$ the TDD RMS sensitivity $s_{f_{\mathrm{d}}}$.

using the perturbation method. The calculation formulae are as follows:

$$
\begin{aligned}
s_{x i} & =\frac{\sigma\left(U_{x i}+\Delta U_{x i}\right)-\sigma\left(U_{x i}\right) / \sigma\left(U_{x i}\right)}{\Delta U_{x i} / U_{x i}}, \\
s_{x} & =\sqrt{\frac{1}{n} \sum_{i=1}^{n}\left|s_{x i}\right|}
\end{aligned}
$$

where $n$ is the number of the intervals, $i$ represents the $i$ th interval, $\Delta U_{x i}$ refers to the incremental value of the system parameter $x$ in the ith interval, and $s_{x}$ is the response sensitivity to the system parameter $x$.
According to the calculated RMS responses based on the system parameters in Table 2, the sensitivity values of each response are obtained by using Equations (31) and (32), as shown in Table 3. To easily observe the differences in sensitivity, the sensitivity values were reordered, as shown in Figure 12. Figure 12(a) shows that $f_{1}$ is the most important influence factor on $\sigma_{\ddot{z}_{1}}$, and $\xi_{0}$ is the weakest influence factor. Other factors are $\xi_{1}, f_{2}, \xi_{2}, r_{2}, r_{0}$, and $f_{0}$ from strong to weak. From Figure 12(b), it can be seen that $\sigma_{\ddot{z}_{2}}$ is most sensitive to $f_{2}$, and the sensitivity is as high as 1.095. $\sigma_{\ddot{z}_{2}}$ is most insensitive to $\xi_{0}$. Other factors are $f_{1}, \xi_{2}, r_{2}, \xi_{1}, f_{0}$, and $r_{0}$ from strong to weak. Figure 12(c) illustrates that the greatest influence factor on $\sigma_{f_{\mathrm{d}}}$ is $f_{1}$, and the minimum influence factor is $\xi_{0}$. Other factors affecting $\sigma_{f_{\mathrm{d}}}$ in an order 
from intensely to slightly are $f_{2}, \xi_{1}, r_{2}, \xi_{2}, f_{0}$, and $r_{0}$. Thus, for the DA, $\xi_{0}$ has the smallest influence on each RMS response, and $f_{0}$ and $r_{0}$ have relatively greater influences.

\section{Conclusions}

This paper compared the vibration isolation performances of the convalescent-wheelchair robot with DA and without DA and conducted the sensitivity analysis of the vibration responses to the important dynamic parameters. The parameters include the following: the mass ratio $r_{0}$ of the DA mass to the wheelchair body mass, the mass ratio $r_{2}$ of the convalescent mass to the wheelchair body mass, the DA natural frequency $f_{0}$, the wheelchair body natural frequency $f_{1}$, the convalescent natural frequency $f_{2}$, the DA system damping ratio $\xi_{0}$, the tire system damping ratio $\xi_{1}$, and the cushion system damping ratio $\xi_{2}$. Some useful conclusions are as follows:

(1) The DA can partly suppress the vibration of the convalescent and the wheelchair body, especially in the resonance area of the wheelchair body. However, the DA cannot successfully improve the tire contact behavior.

(2) The increase of $r_{2}$ is mainly to reduce the values of the wheelchair body RMS acceleration $\sigma_{\ddot{z}_{1}}$ and the convalescent RMS acceleration $\sigma_{\ddot{z}}$. The use of the DA with the larger mass can help to reduce the vibration of the convalescent and the wheelchair. Taking the smaller value of $\xi_{2}$, about 0.2 , is helpful to improve comfort. The ride comfort and the tire grounding safety require that the tire system has the larger value of $\xi_{1}$; however, the operational reliability needs the smaller value of $\xi_{1}$. When the value of $\xi_{0}$ equals 0.2 , the DA system can well give consideration to the comprehensive performance. Adopting a soft cushion to reduce $f_{2}$ is an important measure to improve ride comfort.

(3) $f_{1}$ is the most important influence factor on $\sigma_{\ddot{z}_{1}}$, and $\xi_{0}$ is the weakest influence factor. Other factors are $\xi_{1}, f_{2}, \xi_{2}, r_{2}, r_{0}$, and $f_{0}$ from strong to weak. $\sigma_{\ddot{z}_{2}}$ is most sensitive to $f_{2}$. The greatest influence factor on $\sigma_{f_{\mathrm{d}}}$ is $f_{1}$. For the DA, $\xi_{0}$ has the smallest influence on each RMS response, and $f_{0}$ and $r_{0}$ have relatively greater influences.

\section{Data Availability}

The data used to support the findings of this study are available from the corresponding author upon request.

\section{Conflicts of Interest}

The authors declare that they have no conflicts of interest.

\section{Acknowledgments}

This study was supported by the National Natural Science Foundation of China (no. 61627816) and the National Key R\&D Program of China (no. 2016YFC0701309).

\section{References}

[1] K. T. Kim, H. I. Suk, and S. W. Lee, "Commanding a braincontrolled wheelchair using steady-state somatosensory evoked potential," IEEE Transactions on Neural Systems and Rehabilitation Engineering, vol. 26, no. 3, pp. 654-665, 2018.

[2] D. X. Gong, R. He, G. Y. Zuo, and J. Y. Yu, "Motion mapping in the joint space for the control of the heterogeneous wheelchair-mounted robotic arm," Acta Electronica Sinica, vol. 46, no. 2, pp. 464-472, 2018.

[3] V. T. Nguyen, C. Jayawardena, and I. Ardekani, "A navigation model for side-by-side robotic wheelchairs for optimizing social comfort in crossing situations," Robotics and Autonomous Systems, vol. 100, pp. 27-40, 2018.

[4] V. Travlos, S. Patman, and A. Wilson, "Quality of life and psychosocial well-being in youth with neuromuscular disorders who are wheelchair users: a systematic review," Archives of Physical Medicine and Rehabilitation, vol. 98, no. 5, pp. 1004-1017, 2016.

[5] A. O. Frank and L. H. De Souza, "Clinical features of children and adults with a muscular dystrophy using powered indoor/ outdoor wheelchairs: disease features, comorbidities and complications of disability," Disability and Rehabilitation, vol. 40, no. 9, pp. 1007-1013, 2018.

[6] S. J. Hillman, J. Hollington, N. Crossan, and C. TorresSánchez, "Correlation of ISO 16840-2:2007 impact damping and hysteresis measures for a sample of wheelchair seating cushions," Assistive Technology, vol. 30, no. 2, pp. 77-83, 2018.

[7] K. Brown, H. Flashner, J. L. Mcnitt-Gray, and P. Requejo, "Modeling wheelchair-users undergoing vibrations," Journal of Biomechanical Engineering, vol. 139, no. 9, article 094501, 2017.

[8] Z. Dziechciowski and M. Kromkaszydek, "Vibration transmitted to the human body during the patient's ride in a wheelchair," Archives of Acoustics, vol. 42, no. 1, pp. 137148, 2017.

[9] M. F. Hikmawan and A. S. Nugraha, "Analysis of electric wheelchair passenger comfort with a half car model approach," in Proceeding of 2016 International Conference on Sustainable Energy Engineering and Application: Sustainable Energy for a Better Life (ICSEEA), pp. 76-80, Jakarta, Indonesia, October 2016.

[10] B. F. Wu, P. Y. Chen, and C. H. Lin, "A new criterion of human comfort assessment for wheelchair robots by Q-Learning based accompanist tracking fuzzy controller," International Journal of Fuzzy Systems, vol. 18, no. 6, pp. 1-15, 2016.

[11] S. Y. Wang, C. Y. Sun, and B. G. Wang, "Preliminary study on comfortableness of motorized wheelchair cushion," Journal of Biomedical Engineering, vol. 33, no. 2, pp. 320-324, 2016.

[12] K. Miyawaki and D. Takahashi, "Investigation of whole-body vibration of passenger sitting on wheelchair and of passenger sitting on wheelchair loaded on lifter," in Proceedings of 2016 International Symposium on Micro-NanoMechatronics and Human Science (MHS), Nagoya, Japan, November 2016.

[13] K. H. Su, T. H. Chang, and S. F. Su, "Design of fuzzy-based magnetic suspension vibrator for electric wheelchair," in Proceedings of IEEE 12th International Conference on Networking, Sensing and Control, pp. 586-591, Taipei, Taiwan, April 2015.

[14] A. Ababou, N. Ababou, T. Morsi, and L. Boukhechem, "Test bench for analysis of harmful vibrations induced to wheelchair users," in Proceedings of 7th International Joint 
Conference on Biomedical Engineering Systems and Technologies (BIOSTEC), pp. 147-153, Angers, France, March 2014.

[15] A. S. Kundu, P. K. Lenka, P. K. Lenka, and S. Bhaumik, "Design and performance evaluation of 4 wheeled omni wheelchair with reduced slip and vibration," Procedia Computer Science, vol. 105, pp. 289-295, 2017.

[16] Y. Garcia-Mendenz, J. L. Pearlman, M. L. Boninger, and R. A. Cooper, "Health risks of vibration exposure to wheelchair users in the community," Journal of Spinal Cord Medicine, vol. 36, no. 4, pp. 365-375, 2013.

[17] P.S. Requejo, S. Maneekobkunwong, J. McNitt-Gray et al., "Influence of hand-rim wheelchairs with rear suspension on seat forces and head accelerations during curb descent landings," Journal of Rehabilitation Medicine, vol. 41, no. 6, pp. 459-466, 2009.

[18] D. Brienza, J. Vallely, P. Karg, J. Akins, and A. Gefen, "An MRI investigation of the effects of user anatomy and wheelchair cushion type on tissue deformation," Journal of Tissue Viability, vol. 27, no. 1, pp. 42-53, 2018.

[19] M. Orečný, S. Śtefan, R. Huňady, and F. Želmíra, "Application of a magneto-rheological damper and a dynamic absorber for a suspension of a working machine seat," Procedia Engineering, vol. 96, no. 96, pp. 338-344, 2014.

[20] A. D. Egidio, A. M. D. Leo, and G. Simoneschi, "Effectiveness of mass-damper dynamic absorber on rocking block under one-sine pulse ground motion," International Journal of NonLinear Mechanics, vol. 98, pp. 154-162, 2018.

[21] S. W. Wang, L. L. Zhao, Y. Z. Hu, and F. X. Yang, "Impact responses and parameters sensitivity analysis of electric wheelchairs," Electronics, vol. 7, no. 6, p. 87, 2018.

[22] F. X. Yang, L. L. Zhao, Y. W. Yu, and C. C. Zhou, "Analytical description of ride comfort and optimal damping of cushionsuspension for wheel-drive electric vehicles," International Journal of Automotive Technology, vol. 18, no. 6, pp. 11211129, 2017. 


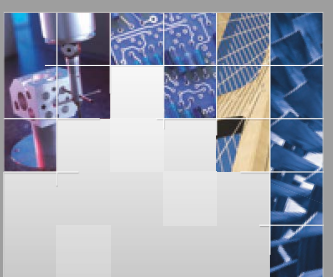

\section{Enfincering}
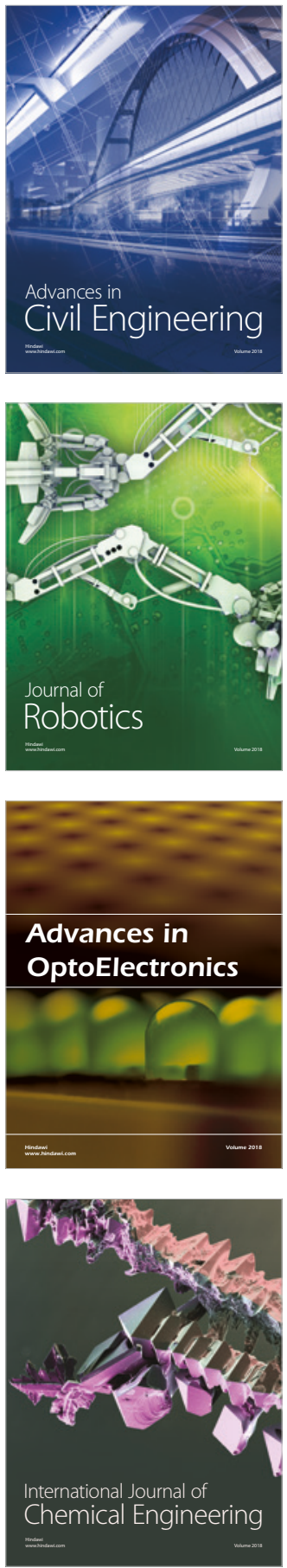

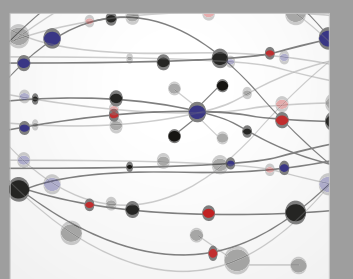

\section{Rotating \\ Machinery}

The Scientific World Journal

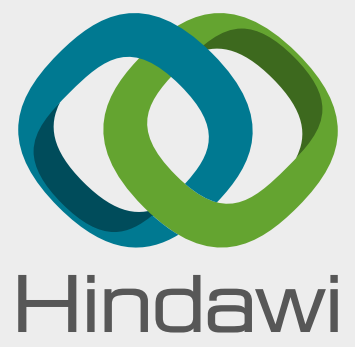

Submit your manuscripts at

www.hindawi.com
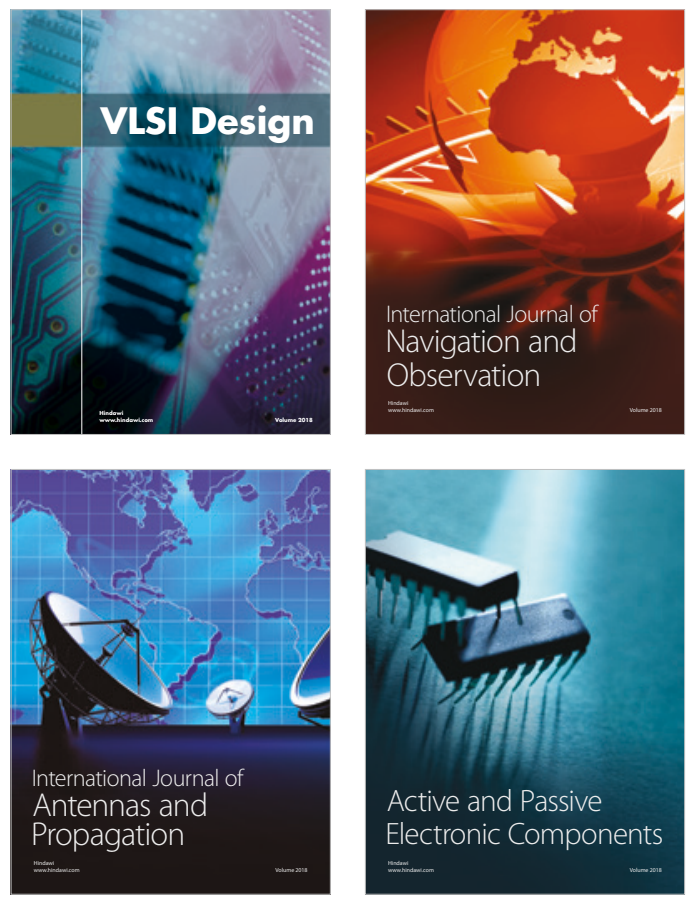
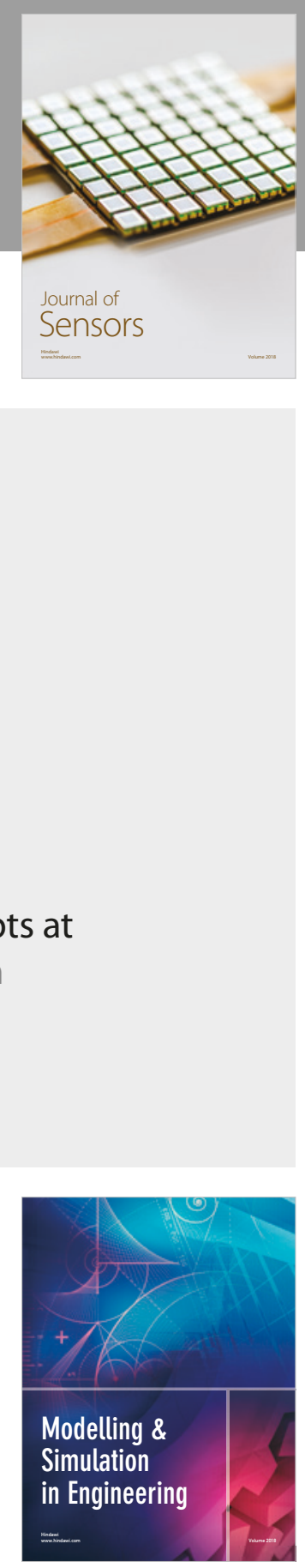

\section{Advances \\ Multimedia}
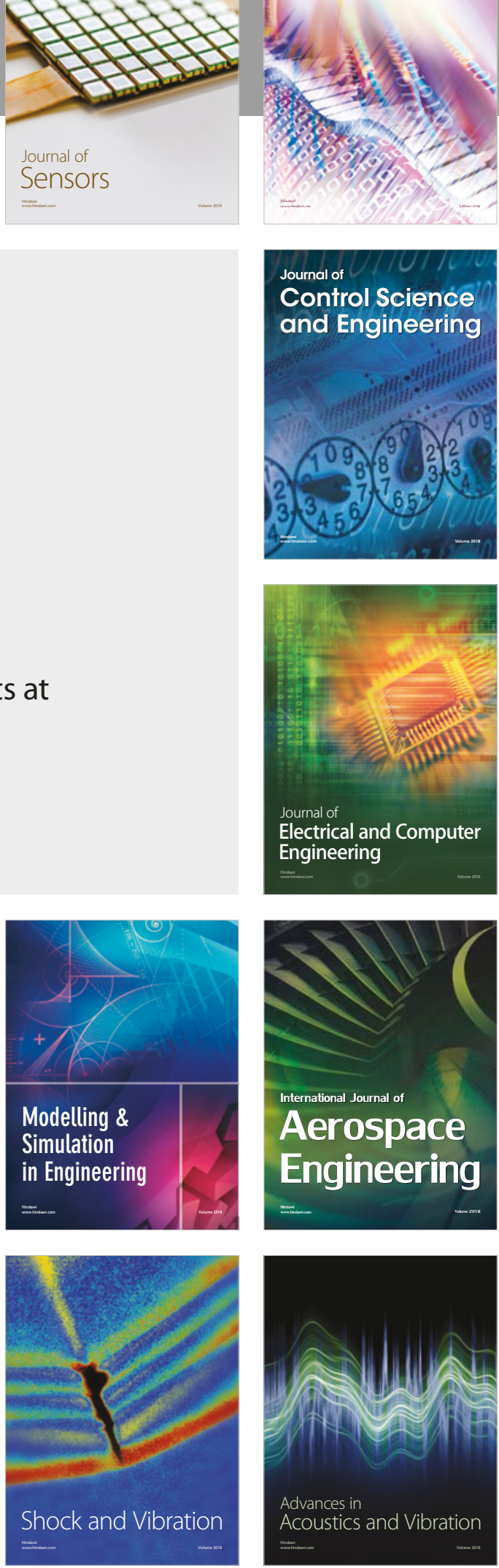